JOURNAL OF THE

AMERICAN MATHEMATICAL SOCIETY

Volume 18, Number 2, Pages 291-312

S 0894-0347(04)00474-6

Article electronically published on December 7, 2004

\title{
GREEN CURRENTS FOR HOLOMORPHIC AUTOMORPHISMS OF COMPACT KÄHLER MANIFOLDS
}

\author{
TIEN-CUONG DINH AND NESSIM SIBONY
}

\section{INTRODUCTION}

Let $(X, \omega)$ be a compact Kähler manifold of dimension $k$. Let $f$ be a holomorphic automorphism of $X$. Our purpose is to introduce invariant positive closed currents for $f$ and to use them in order to construct dynamically interesting invariant measures.

More precisely we want to construct positive closed $(s, s)$-currents $T$ satisfying a functional equation

$$
f^{*} T=\lambda T, \quad \lambda>1,
$$

and some additional regularity properties. Very likely, the currents $T$ will describe the distribution of invariant manifolds of codimension $s$ corresponding to the smallest Lyapounov exponents.

Let $d_{p}$ denote the dynamical degree of order $p$ of $f$. It describes the growth under iteration of the volume of $p$-dimensional manifolds. When $d_{1}>1$, it is natural to introduce first a positive closed $(1,1)$-current as

$$
T_{1}:=\lim _{n \rightarrow \infty} \frac{\left(f^{n}\right)^{*} \omega}{d_{1}^{n}}
$$

Unfortunately, this limit does not always exist, as holomorphic automorphisms of tori show.

However, for Hénon maps in $\mathbb{C}^{2}$ or for algebraically stable meromorphic self-maps of $\mathbb{P}^{k}$ the limit exists and was studied extensively. See the introduction of [1, 35. for historical comments. The limit $T_{1}$ is called the Green current. For holomorphic endomorphisms of $\mathbb{P}^{k}$, the self-intersection $T_{1}^{k}$ is well defined and gives an invariant measure.

In the context of polynomial automorphisms $f$ of $\mathbb{C}^{2}$ having positive entropy, the second author has defined, using the intersection of the Green currents of $f$ and $f^{-1}$, an invariant probability measure which turned out to be dynamically interesting. It, as well as the Green current, was extensively studied by Bedford-Lyubich-Smillie [2, 1] and by Fornæss and the second author [22, 23].

Cantat 6 has adapted some of the constructions from the study of Hénon maps to the context of automorphisms of K3 surfaces. The striking fact in this case is the existence of automorphisms of positive entropy for some K3 surfaces [33 66, 34].

Received by the editors November 20, 2003.

2000 Mathematics Subject Classification. Primary 37F10, 32H50, 32Q15, 32U40.

Key words and phrases. Green current, equilibrium measure, mixing.

(C)2004 American Mathematical Society Reverts to public domain 28 years from publication 
Given a holomorphic automorphism or, more generally, a bimeromorphic selfmap of a compact Kähler manifold of dimension $k \geq 3$, constructing interesting invariant measures is a delicate process. The case of some classes of polynomial automorphisms of $\mathbb{C}^{k}$ is considered in [35, 30]. The same difficulty arises for meromorphic self-maps whose topological degree is not the largest of the dynamical degrees; see [21, 28, 14]. In this case, the cohomology class $\left[T_{1}\right]^{k}$ is zero in the cohomology group $\mathcal{H}^{k, k}(X, \mathbb{R})$. Hence we cannot construct an invariant measure as the self-intersection of the Green current $T_{1}$.

In this paper, we focus on the question of constructing invariant currents and invariant measures for automorphisms of compact Kähler manifolds. We consider first automorphisms to avoid some technicalities. Some of the results can be extended to holomorphic maps, to non-singular correspondences and to birational maps [19].

When $d_{1}>1$, we consider the convex cone $\Gamma_{1}$ of positive closed $(1,1)$-currents $T_{1}$ with bounded potentials such that $f^{*} T_{1}=d_{1} T_{1}$. We will show that this cone is closed, finite dimensional and not reduced to zero. Moreover, every current in $\Gamma_{1}$ has a Hölder continuous potential. An element of $\Gamma_{1}$ is called a Green current of bidegree $(1,1)$ (see 6, 28, for the case of surfaces).

To construct invariant currents of higher bidegree, we start with a positive closed current $T$ of bidegree $(s, s)$ satisfying

$$
f^{*} T=\lambda_{T} T, \lambda_{T}>0 .
$$

We introduce the cone $\Gamma(T)$ of positive closed $(s+1, s+1)$-currents $T \wedge S$ such that $T \wedge f^{*} S=\lambda_{1}(T) T \wedge S$ where $S$ is a closed (not necessarily positive) (1,1)-current with a continuous potential and

$$
\lambda_{1}(T):=\lim _{n \rightarrow \infty}\left(\int T \wedge\left(f^{n}\right)^{*} \omega \wedge \omega^{k-s-1}\right)^{1 / n} .
$$

The number $\lambda_{1}(T)$ appears as a dynamical degree with respect to $T$. When $\lambda_{1}(T)>$ 1 , we show that $\Gamma(T)$ is a closed, finite dimensional, convex cone, not reduced to zero. This requires the introduction of cohomology groups with respect to $T$. We also prove that $\Gamma(T)$ is unchanged if we consider only currents $S$ with Hölder continuous potentials. In this approach, we use an inductive procedure, and at the step $s$ we replace the complex manifold $X$ by the $(s, s)$-current $T$.

When $d_{1}$ is strictly larger than the other dynamical degrees of $f$, using $f$ and $f^{-1}$, we can construct invariant positive currents as an intersection of closed $(1,1)$ currents. We first construct the current $T_{1}$ such that $f^{*} T_{1}=d_{1} T_{1}$ as above. We have $f_{*} T_{1}=d_{1}^{-1} T_{1}$. We then construct $(1,1)$-currents $S_{i}, 1 \leq i \leq k-1$, with Hölder continuous potentials such that the currents $T_{1} \wedge S_{1} \wedge \ldots \wedge S_{i}$ are positive, closed and satisfy

$$
f_{*}\left(T_{1} \wedge S_{1} \wedge \ldots \wedge S_{i}\right)=c_{i} T_{1} \wedge S_{1} \wedge \ldots \wedge S_{i} \text { for some } c_{i}>0 .
$$

In this case, we can show, at each step, that for the automorphism $f^{-1}$, the dynamical degree $\lambda_{1}\left(T_{1} \wedge S_{1} \wedge \ldots \wedge S_{i}\right)$, with respect to the current $T_{1} \wedge S_{1} \wedge \ldots \wedge S_{i}$, is strictly larger than 1 . The measure $\mu:=T_{k}$ is invariant, mixing and gives no mass to pluripolar sets and to sets of small Hausdorff dimension.

In the general case, the method breaks down because the hypothesis on $\lambda_{1}(T)$ is not easy to check. Therefore, we introduce a second method for constructing 
directly an invariant current of bidegree $(s, s)$, under the assumption $d_{s}>d_{s-1}$. The current we construct is $\mathrm{PB}$ and satisfies

$$
f^{*} T=d_{s} T \text {. }
$$

Note that if a current $T$ is $\mathrm{PB}$, it is weakly $\mathrm{PB}$, i.e. q.p.s.h. functions are integrable with respect to the trace measure $T \wedge \omega^{k-s}$ (see Section 2.1 for details). The method uses a $\mathrm{dd}^{\mathrm{c}}$-resolution as already used by the authors in various contexts $13,16,11$. We have to use complex analysis, i.e. estimates for the solutions of the $\mathrm{dd}^{\mathrm{c}}$-equation (Proposition 2.1). This permits us to control the growth of $\left(f^{n}\right)_{*} \Phi$, where $\Phi$ is a test form. The delicate point in the construction is to obtain a PB current. See also 20] for another method.

The method permits us to prove that $T$ is almost extremal, i.e. $T$ belongs to a finite dimensional extremal face of the cone of positive closed currents.

To construct an invariant measure $\mu$, we assume that the dynamical degrees of the automorphism $f$ are distinct. Then the Khovanskii-Tessier-Gromov concavity theorem implies the existence of an $m$ such that

$$
1<d_{1}<\cdots<d_{m}>d_{m+1}>\cdots>d_{k}=1
$$

The measure $\mu$ is then obtained using the first method for $f^{-1}$ but starting with $T_{m}$. Therefore, $\mu$ is a wedge product of $T_{m}$ with closed $(1,1)$-currents with Hölder continuous potentials. We can choose $T_{m}$ so that $\mu$ is mixing and has positive Hausdorff dimension. Observe that according to Yomdin-Gromov [38, 26, 17, 18] the topological entropy $\mathrm{h}(f)$ of $f$ is equal to max $\log d_{i}$. Hence, the PB current $T_{m}$ satisfies $f^{*} T_{m}=\exp (\mathrm{h}(f)) T_{m}$.

The classes of PC and PB currents, which we introduce, are of interest since they allow us to define the product of currents of higher bidegree. We will come back to this subject in a furture work.

Many questions concerning Green currents and the measure $\mu$ are not studied here, for instance: distribution of periodic points with respect to $\mu$, entropy of $\mu$, approximation of the Green currents by stable leaves, ... If $X$ is projective, the first author has proved that $T_{m}$ is weakly laminar 12 .

The classification of manifolds of dimension $\geq 3$ with automorphisms of positive entropy is also an interesting problem. In dimension 2, many examples are given in [33, 6, 34]. Mazur's examples can be extended to dimension $\geq 3$.

Mazur's examples. Let $X$ be a smooth hypersurface of degree 2 of $\mathbb{P}^{1, k+1}:=$ $\mathbb{P}^{1} \times \cdots \times \mathbb{P}^{1}(k+1$ factors $)$. Let $\pi_{i}, 1 \leq i \leq k+1$, denote the $k+1$ projections of $X$ on the product of $k$ factors of $\mathbb{P}^{1, k+1}$. Assume that all $\pi_{i}$ are finite. Then, each fiber of $\pi_{i}$ contains exactly two points $z, z^{\prime}$. We can define an involution $\tau_{i}$ by $z \mapsto z^{\prime}$. The group generated by $\tau_{i}$ contains dynamically interesting automorphisms.

We can construct other examples by taking products of manifolds or the projectivization of their tangent bundle. The dynamics of these examples is however non-trivial. It is used in [12] to get information on laminarity of currents. Examples on tori explain some of the difficulties that we have to overcome in the general case. Our results extend to non-singular correspondences (see [32, p. 337] and [7, 37, 11, 16] for definitions and examples). 


\section{Currents And Groups of COHOMOlogy}

A holomorphic automorphism $f$ of $X$ induces an invertible linear self-map on groups of cohomology. We will use this action of $f$ in order to construct invariant currents. We introduce classes of currents with some regularity properties.

We will write $u_{n} \simeq v_{n}$ for $\lim u_{n} / v_{n}=1$. The notation $u_{n} \lesssim v_{n}$ means $\lim \sup \left|u_{n} / v_{n}\right|<+\infty$, and the notation $u_{n} \sim v_{n}$ means $u_{n} \lesssim v_{n}$ and $v_{n} \lesssim u_{n}$, with the convention that $0 \simeq 0,0 \lesssim 0$ and $0 \sim 0$. For $(x, y)$ and $\left(x^{\prime}, y^{\prime}\right)$ in $\mathbb{R}^{2}$, we write $(x, y) \leq\left(x^{\prime}, y^{\prime}\right)$ if either $x<x^{\prime}$ or if $x=x^{\prime}$ and $y \leq y^{\prime}$. The sign \|\| denotes either the mass of currents, the norm of a vector, or the norm of a linear operator. The sign [ ] denotes a cohomology class of a closed current.

2.1. PC, PB and weakly PB currents. We refer to the survey by Demailly 8 for the basics on currents in complex analysis. Demailly's survey on Hodge theory [9] is also useful. We however recall a few facts.

When $(X, \omega)$ is a compact Kähler manifold of dimension $k$, a current $T$ of bidegree $(s, s)$ is a continuous linear form on $\mathcal{D}^{k-s, k-s}(X)$ - the space of smooth forms of bidegree $(k-s, k-s)$. In a coordinate chart, $T$ can be expressed as a differential $(s, s)$-form with distribution coefficients. A $(k-s, k-s)$-form $\Phi$ is weakly positive if at every point $z \in X$

$$
\Phi \wedge i \alpha_{1} \wedge \bar{\alpha}_{1} \wedge \ldots \wedge i \alpha_{s} \wedge \bar{\alpha}_{s} \geq 0
$$

for every (1,0)-form $\alpha_{j}$ of $X$. The current $T$ is called (strongly) positive if $\langle T, \Phi\rangle \geq 0$ for every weakly positive test form $\Phi$. The space of currents is given the weak topology of currents. In particular, positive currents are currents of order zero. The trace measure $\sigma_{T}$ associated to a positive current $T$ is defined as $\sigma_{T}:=\frac{1}{(k-s) !} T \wedge$ $\omega^{k-s}$. The measure $\sigma_{T}$ is positive and the coefficients of $T$ in a chart are measures which are dominated by $c \sigma_{T}, c>0$. We will denote by $\|T\|$ the mass $\int_{X} T \wedge \omega^{k-s}$ of $T$.

The calculus on differential forms extends to currents, except for the pullback by a holomorphic map, which is not a submersion. It is always delicate to define the wedge product of two currents. However when $u \in \mathrm{L}^{1}\left(\sigma_{T}\right)$, for example if $u$ is bounded, one can define $\operatorname{dd}^{c} u \wedge T:=\operatorname{dd}^{c}(u T)$. The continuity properties of this operator depend on the properties of $u[8]$. Recall that $\mathrm{d}=\partial+\bar{\partial}, \mathrm{d}^{\mathrm{c}}=\frac{1}{2 i \pi}(\partial-\bar{\partial})$ and that $\mathrm{dd}^{\mathrm{c}}=\frac{i}{\pi} \partial \bar{\partial}$ is a real operator.

Let $T$ be a positive closed $(s, s)$-current, and let $S$ be a closed $(1,1)$-current not necessarily positive. Since $X$ is Kähler, by Hodge theory, we can write $S=\alpha+\operatorname{dd}^{\mathrm{c}} u$, where $\alpha$ is a smooth form cohomologous to $S$ and $u$ is a $(0,0)$-current. We say that $u$ is a potential of $S$. Observe that two potentials of $S$ differ by a smooth function. When $u$ is a $\nu$-Hölder continuous (resp. continuous or bounded) function, we say that $S$ has a $\nu$-Hölder continuous (resp. a continuous or bounded) potential. It is clear that this is independent of the choice of $\alpha$. For a current $S$ with a continuous potential, we can define $T \wedge S$ by $T \wedge S:=T \wedge \alpha+\operatorname{dd}^{c}(u T)$. When $S$ is positive, we can choose $u$ upper semi-continuous. In this case, if $u$ is bounded, we can define $T \wedge S$ in the same way.

A real $(p, p)$-current $\Phi$ on $X$ is called $D S H$ if $\Phi=\Phi_{1}-\Phi_{2}$ and $\mathrm{dd}^{\mathrm{c}} \Phi_{i}=\Omega_{i}^{+}-\Omega_{i}^{-}$ where $\Phi_{i}$ are negative currents, and $\Omega_{i}^{ \pm}$are positive closed currents. We define the DSH-norm by

$$
\|\Phi\|_{\mathrm{DSH}}:=\inf \left\{\left\|\Phi_{1}\right\|+\left\|\Phi_{2}\right\|+\left\|\Omega_{1}^{+}\right\|+\left\|\Omega_{2}^{+}\right\|, \Phi_{i}, \Omega_{i}^{ \pm} \text {as above }\right\} .
$$


Observe that $\left\|\Omega_{i}^{+}\right\|=\left\|\Omega_{i}^{-}\right\|$, and we can choose $\Phi_{i}$ and $\Omega_{i}^{ \pm}$such that $\|\Phi\|_{\mathrm{DSH}}=$ $\left\|\Phi_{1}\right\|+\left\|\Phi_{2}\right\|+\left\|\Omega_{1}^{+}\right\|+\left\|\Omega_{2}^{+}\right\|$. Denote by $\operatorname{DSH}^{p}(X)$ the space of DSH $(p, p)$-currents. This is our space of test currents. A current $\Phi$ is in $\operatorname{DSH}^{0}(X)$ if and only if it is a difference of q.p.s.h. functions.

Recall that an $\mathrm{L}^{1}$ function $\varphi: X \rightarrow \mathbb{R} \cup\{-\infty\}$ is quasi-plurisubharmonic (q.p.s.h. for short) if it is upper semi-continuous and $\operatorname{dd}^{c} \varphi \geq-c \omega, c>0$, in the sense of currents. A set $E \subset X$ is pluripolar if it is contained in the pole set $\{\varphi=-\infty\}$ of a q.p.s.h. function $\varphi$.

A topology on $\operatorname{DSH}^{p}(X)$ is defined in the following way: $\Phi^{(n)} \rightarrow \Phi$ in $\operatorname{DSH}^{p}(X)$ if $\Phi^{(n)} \rightarrow \Phi$ weakly and if $\left\|\Phi^{(n)}\right\|_{\text {DSH }}$ is bounded.

Let $T$ be a current of bidegree $(s, s)$ and of zero order. We say that $T$ is $P C$ if it can be extended to a continuous linear form on $\mathrm{DSH}^{k-s}(X)$, and we write $\langle T, \Phi\rangle$ for the value of this linear form on $\Phi \in \mathrm{DSH}^{k-s}(X)$. In [18, we proved that every current in $\mathrm{DSH}^{k-s}(X)$ can be approximated by smooth forms. Then, the extension of $T$ is unique. Moreover, when $\Phi$ is continuous, $\langle T, \Phi\rangle$ coincides with the usual integral [18, Prop. 4.6].

We say that $T$ is $P B$ if there exists a constant $c_{T}>0$ such that

$$
|\langle T, \Phi\rangle| \leq c_{T}\|\Phi\|_{\mathrm{DSH}} \text { for every DSH continuous }(k-s, k-s) \text {-form } \Phi .
$$

The current $T$ is called weakly $P B$ if

$$
\left|\left\langle T, \varphi \omega^{k-s}\right\rangle\right|<+\infty \text { for every q.p.s.h. function } \varphi \text { on } X \text {. }
$$

Observe that $T$ is weakly PB if and only if the measure $T \wedge \omega^{k-s}$ is weakly PB. If a positive current $T$ is (weakly) $\mathrm{PB}$, then every positive current $T^{\prime}$ such that $T^{\prime} \leq T$ is also (weakly) PB. We can show that if $T$ is a positive weakly PB current, then $\left|\left\langle T, \varphi \omega^{k-s}\right\rangle\right| \leq c_{T}\left(1+\|\varphi\|_{\mathrm{L}^{1}}\right)$ for some constant $c_{T}>0$ and for $\varphi$ q.p.s.h. such that $\operatorname{dd}^{\mathrm{c}} \varphi \geq-\omega$ (see [16] or Proposition 2.2).

In 13, we showed that a positive measure $\mu$ on a Riemann surface admits locally a bounded potential if and only if locally p.s.h. functions are $\mu$-integrable. This justifies the term PB (see also Proposition 2.2).

The following result is useful in constructing PC and PB currents. It supplies the fact that when $\Omega^{-}$is a smooth form cohomologous to a positive closed current $\Omega^{+}$, we cannot find, in general, a negative form $\Phi$ solving $\operatorname{dd}^{\mathrm{c}} \Phi=\Omega^{+}-\Omega^{-}$. A counterexample can be found in [4].

Proposition 2.1. There exists a constant $A>0$ so that for every pair of positive closed $(k-s+1, k-s+1)$-currents $\Omega^{ \pm}$on $X$ with $\left[\Omega^{+}\right]=\left[\Omega^{-}\right]$, there are $\mathrm{L}^{1}$ negative $(k-s, k-s)$-forms $\Phi^{ \pm}$such that

$$
\operatorname{dd}^{\mathrm{c}} \Phi^{+}-\mathrm{dd}^{\mathrm{c}} \Phi^{-}=\Omega^{+}-\Omega^{-} \text {and }\left\|\Phi^{ \pm}\right\|_{\mathrm{DSH}} \leq A\left\|\Omega^{+}\right\| .
$$

Moreover, the DSH currents $\Phi^{ \pm}$depend continuously on $\Omega^{ \pm}$. If $\Omega^{ \pm}$are continuous, then $\Phi^{ \pm}$are continuous.

Proof. Analogous problems are considered in [18] and other aspects of the following computation are detailed there. By Hodge theory [25], we have

$$
\mathcal{H}^{k, k}(X \times X, \mathbb{C}) \simeq \sum_{\substack{p+p^{\prime}=k \\ q+q^{\prime}=k}} \mathcal{H}^{p, q}(X, \mathbb{C}) \otimes_{\mathbb{C}} \mathcal{H}^{p^{\prime}, q^{\prime}}(X, \mathbb{C}) .
$$


Hence, if $\Delta$ is the diagonal of $X \times X$, there exists a smooth real $(k, k)$-form $\alpha(x, y)$ on $X \times X$, cohomologous to $[\Delta]$ with $\mathrm{d}_{x} \alpha=\mathrm{d}_{y} \alpha=0$. Following Bost-GilletSoulé [24, 4], we can construct a $(k-1, k-1)$-form $K(x, y)$ on $X \times X$ such that $\operatorname{dd}^{c} K=[\Delta]-\alpha$. We recall the construction.

Let $\pi: \widehat{X \times X} \longrightarrow X \times X$ be the blow-up of $X \times X$ along $\Delta$. It follows from a theorem of Blanchard [3] that $\widehat{X \times X}$ is a Kähler manifold. Let $\widehat{\Delta}:=\pi^{-1}(\Delta)$ be the exceptional hypersurface. Choose a negative q.p.s.h. function $\widehat{\varphi}$ on $\widehat{X \times X}$ such that $\gamma:=-\operatorname{dd}^{\mathrm{c}} \widehat{\varphi}+[\widehat{\Delta}]$ is a smooth form cohomologous to $[\widehat{\Delta}]$. We can choose 24, 1.3.6] a smooth closed real $(k-1, k-1)$-form $\eta$ on $\widehat{X \times X}$ such that $\pi^{*} \alpha$ is cohomologous to $[\widehat{\Delta}] \wedge \eta$, hence to $\gamma \wedge \eta$. It follows that there is a smooth real $(k-1, k-1)$-form $\beta$ such that $\operatorname{dd}^{\mathrm{c}} \beta=-\gamma \wedge \eta+\pi^{*} \alpha$. Define

$$
K(x, y):=\pi_{*}(\widehat{\varphi} \eta-\beta) .
$$

We have

$$
\operatorname{dd}^{\mathrm{c}} K(x, y)=\pi_{*}\left([\widehat{\Delta}] \wedge \eta-\pi^{*} \alpha\right)=\pi_{*}([\widehat{\Delta}] \wedge \eta)-\alpha .
$$

The $(k, k)$-current $\pi_{*}([\widehat{\Delta}] \wedge \eta)$ is closed, of order zero and supported on $\Delta$. Hence, it is a multiple of $[\Delta]$. Moreover, $\pi_{*}([\widehat{\Delta}] \wedge \eta)$ is cohomologous to $[\alpha]=[\Delta]$. It follows that $\pi_{*}([\widehat{\Delta}] \wedge \eta)=[\Delta]$ and $\operatorname{dd}^{\mathrm{c}} K=[\Delta]-\alpha$.

Since the forms $\eta$ and $\beta$ are smooth, we can write $\eta:=\eta^{+}-\eta^{-}$and $\beta:=\beta^{+}-\beta^{-}$ with positive closed smooth forms $\eta^{ \pm}$and negative smooth forms $\beta^{ \pm}$. Define $K^{ \pm}:=$ $\pi_{*}\left(\widehat{\varphi} \eta^{ \pm}+\beta^{\mp}\right)$. These forms are negative and we have $K=K^{+}-K^{-}$. Moreover, there exist constants $c^{ \pm}$with $c^{+}-c^{-}=1$ and closed real $(k, k)$-forms $\Theta^{ \pm}$on $X \times X$ such that $\mathrm{dd}^{c} K^{ \pm}=\Theta^{ \pm}+c^{ \pm}[\Delta]$. Let $|x-y|$ denote the distance between two points $x$ and $y$ of $X$ with respect to the Kähler metric on $X$. One can check that $K^{ \pm}, \Theta^{ \pm}$ are smooth on $X \times X \backslash \Delta$ and

$$
K^{ \pm}(x, y)=\mathrm{O}\left(|x-y|^{2-2 k} \log |x-y|\right), \quad \Theta^{ \pm}=\mathrm{O}\left(|x-y|^{2-2 k}\right)
$$

when $(x, y) \rightarrow \Delta$. This allows us to define

$$
\begin{aligned}
& \Phi^{+}(x):=\int_{y \in X} K^{+}(x, y) \wedge \Omega^{+}(y)+\int_{y \in X} K^{-}(x, y) \wedge \Omega^{-}(y), \\
& \Phi^{-}(x):=\int_{y \in X} K^{+}(x, y) \wedge \Omega^{-}(y)+\int_{y \in X} K^{-}(x, y) \wedge \Omega^{+}(y) .
\end{aligned}
$$

Since $\mathrm{dd}^{\mathrm{c}} K=[\Delta]-\alpha, \mathrm{d}_{y} \alpha=0$, and $\Omega^{+}-\Omega^{-}$is exact, we have

$$
\begin{aligned}
\mathrm{dd}^{\mathrm{c}} \Phi^{+}(x) & -\mathrm{dd}^{\mathrm{c}} \Phi^{-}(x)=\int_{y \in X}\left(\mathrm{dd}^{\mathrm{c}}\right)_{x} K(x, y) \wedge\left(\Omega^{+}(y)-\Omega^{-}(y)\right) \\
= & \int_{y \in X} \mathrm{dd}^{\mathrm{c}} K(x, y) \wedge\left(\Omega^{+}(y)-\Omega^{-}(y)\right) \\
= & \Omega^{+}(x)-\Omega^{-}(x)-\int_{y \in X} \alpha(x, y) \wedge\left(\Omega^{+}(y)-\Omega^{-}(y)\right) \\
= & \Omega^{+}(x)-\Omega^{-}(x) .
\end{aligned}
$$

The description of the singularities of $K^{ \pm}$implies that $\left\|\Phi^{ \pm}\right\|_{\mathrm{L}^{1}} \lesssim\left\|\Omega^{+}\right\|$, that $\Phi^{ \pm}$ depend continuously on $\Omega^{ \pm}$and that $\Phi^{ \pm}$are continuous when $\Omega^{ \pm}$are continuous. We can also write $\Theta^{ \pm}$as differences of positive closed forms, smooth on $X \times X \backslash \Delta$, with singularities of order $\mathrm{O}\left(|x-y|^{2-2 k}\right)$. It follows that $\left\|\Phi^{ \pm}\right\|_{\mathrm{DSH}} \lesssim\left\|\Omega^{+}\right\|$. 
Proposition 2.2. Let $T$ be a positive $(s, s)$-current on $X$. If $T$ is $P C$, then $T$ is $P B$. If $T$ is $P B$, then it is weakly $P B$. A positive measure $\mu$ on $X$ is $P B$ if and only if it is weakly $P B$.

Proof. Let $\Phi_{n}$ be continuous forms with $\left\|\Phi_{n}\right\|_{\mathrm{DSH}}=1$. If $c_{n}:=\left|\left\langle T, \Phi_{n}\right\rangle\right|$ tend to $+\infty$, then $c_{n}^{-1} \Phi_{n}$ converge to 0 in $\operatorname{DSH}^{k-s}(X)$, and $\left|\left\langle T, c_{n}^{-1} \Phi_{n}\right\rangle\right|$ converge to 1 . Hence, $T$ is not PC.

Assume now that $T$ is PB. Let $\varphi$ be a strictly negative q.p.s.h. function on $X$ such that $\operatorname{dd}^{\mathrm{c}} \varphi \geq-\omega$. We have to show that $\left\langle T, \varphi \omega^{k-s}\right\rangle>-\infty$. Following a theorem of Demailly [10], there exists a decreasing sequence of smooth negative functions $\left(\varphi_{n}\right)$ with limit $\varphi$, which satisfy $\operatorname{dd}^{\mathrm{c}} \varphi_{n} \geq-c_{X} \omega$ where $c_{X}>0$ is a constant. We then have

$$
\left|\left\langle T, \varphi \omega^{k-s}\right\rangle\right|=\lim \left|\left\langle T, \varphi_{n} \omega^{k-s}\right\rangle\right| \lesssim \limsup \left\|\varphi_{n} \omega^{k-s}\right\|_{\mathrm{DSH}} \lesssim\|\varphi\|_{\mathrm{L}^{1}}+1 .
$$

Hence $T$ is weakly PB.

Now, assume that $\mu$ is a weakly $\mathrm{PB}$ probability measure. We show that it is PB. If not, there would exist continuous functions $\varphi_{n}$ such that $\left\|\varphi_{n}\right\|_{\text {DSH }}=1$ and $\left\langle\mu, \varphi_{n}\right\rangle \geq n^{3}$. We can write $\operatorname{dd}^{\mathrm{c}} \varphi_{n}=\Omega_{n}^{+}-\Omega_{n}^{-}$where $\Omega_{n}^{ \pm}$are positive closed $(1,1)$-currents such that $\left\|\Omega_{n}^{ \pm}\right\| \leq 1$. Since the set of such currents is compact, there exist smooth forms $\Omega_{n}$, cohomologous to $\Omega_{n}^{ \pm}$, such that $\Omega_{n} \leq c \omega$, where $c>0$ is a constant independent of $n$. There exist q.p.s.h. functions $\varphi_{n}^{ \pm}$satisfying $\operatorname{dd}^{\mathrm{c}} \varphi_{n}^{ \pm}=\Omega_{n}^{ \pm}-\Omega_{n}$ and $\max _{X} \varphi_{n}^{ \pm}=0$. The family of q.p.s.h. functions $\psi$ such that $\max _{X} \psi=0$ and $\mathrm{dd}^{\mathrm{c}} \psi \geq-c \omega$ is compact in $\mathrm{L}^{1}(X)$. Hence there is a constant $A>0$ such that $\left\|\varphi_{n}^{ \pm}\right\|_{\mathrm{DSH}} \leq A$. Define $c_{n}:=\varphi_{n}-\varphi_{n}^{+}+\varphi_{n}^{-}$. We have $\mathrm{dd}^{\mathrm{c}} c_{n}=0$. Hence $c_{n}$ is a constant and $\left|c_{n}\right| \lesssim\left\|\varphi_{n}\right\|_{\mathrm{L}^{1}}+\left\|\varphi_{n}^{+}\right\|_{\mathrm{L}^{1}}+\left\|\varphi_{n}^{-}\right\|_{\mathrm{L}^{1}} \leq 1+2 A$. We then deduce, since $\varphi_{n}^{+} \leq 0$, that

$$
\left\langle\mu, \varphi_{n}^{-}\right\rangle=-\left\langle\mu, \varphi_{n}\right\rangle+c_{n}+\left\langle\mu, \varphi_{n}^{+}\right\rangle \lesssim-n^{3}+1+2 A .
$$

It follows that $\langle\mu, \varphi\rangle=-\infty$ if $\varphi:=\sum n^{-2} \varphi_{n}^{-}$. This is a contradiction because the series $\sum n^{-2} \varphi_{n}^{-}$converges to a q.p.s.h. function.

2.2. Some properties of linear maps. Recall that a Jordan block $J_{\lambda, m}$ is a square matrix $\left(a_{i, j}\right)_{1 \leq i, j \leq m}$ such that $a_{i j}=\lambda$ if $i=j, a_{i j}=1$ if $j=i+1$ and $a_{i j}=0$ otherwise. If $\lambda \neq 0$, the entry of index $(1, m)$ of $J_{\lambda, m}^{n}$ is equal to $\left(\begin{array}{c}n \\ m-1\end{array}\right) \lambda^{n-m+1}$. This is the only entry of order $n^{m-1}|\lambda|^{n}$; the other ones have order at most $n^{m-2}|\lambda|^{n}$. We have

$$
\left\|J_{\lambda, m}^{n}\right\| \sim\left(\begin{array}{c}
n \\
m-1
\end{array}\right)|\lambda|^{n-m+1} \sim n^{m-1}|\lambda|^{n} .
$$

The eigenspace of $J_{\lambda, m}$ associated to the unique eigenvalue $\lambda$ is a complex line.

If $E, E^{\prime}$ are real (resp. complex) vector spaces, denote by $\operatorname{End}\left(E, E^{\prime}\right)$ the space of $\mathbb{R}$-linear (resp. $\mathbb{C}$-linear) maps from $E$ onto $E^{\prime}$. When $E$ and $E^{\prime}$ are real vector spaces, we have

$$
\operatorname{End}\left(E, E^{\prime}\right) \otimes_{\mathbb{R}} \mathbb{C}=\operatorname{End}\left(E \otimes_{\mathbb{R}} \mathbb{C}, E^{\prime} \otimes_{\mathbb{R}} \mathbb{C}\right) .
$$

Hence, we identify $\operatorname{End}\left(E, E^{\prime}\right)$ with a real vector subspace of $\operatorname{End}\left(E \otimes_{\mathbb{R}} \mathbb{C}, E^{\prime} \otimes_{\mathbb{R}} \mathbb{C}\right)$.

Consider a complex space $E$ and an invertible linear map $\Lambda \in \operatorname{End}(E, E)$. The space $E$ is the direct sum of the invariant complex subspaces $E=E_{1} \oplus \cdots \oplus E_{r}$ with $\operatorname{dim} E_{i}=m_{i}$. The restriction of $\Lambda$ to $E_{i}$ is defined by a Jordan block $J_{\lambda_{i}, m_{i}}$. We can assume that $\left(\left|\lambda_{1}\right|, m_{1}\right) \geq \cdots \geq\left(\left|\lambda_{r}\right|, m_{r}\right)$. Let $F_{i}$ denote the eigenspace of 
$\Lambda_{\mid E_{i}}$. It is a complex line. Let $E_{i}^{\prime}$ be the hyperplane generated by the first $\left(m_{i}-1\right)$ vectors of the basis of $E_{i}$ associated to the Jordan form.

We say that $J_{\lambda_{i}, m_{i}}$ is dominant if $\left(\left|\lambda_{i}\right|, m_{i}\right)=\left(\left|\lambda_{1}\right|, m_{1}\right)$. In this case, we say that $\lambda_{i}$ is a dominant eigenvalue of $\Lambda$. Assume that $J_{\lambda_{1}, m_{1}}, \ldots, J_{\lambda_{\nu}, m_{\nu}}$ are the dominant Jordan blocks. It is clear that $\left\|\Lambda^{n}\right\| \sim n^{m_{1}-1}\left|\lambda_{1}\right|^{n}$ and that for any vector $v \notin E_{1}^{\prime} \oplus \cdots \oplus E_{\nu}^{\prime} \oplus E_{\nu+1} \oplus \cdots \oplus E_{r}$, we have $\left\|\Lambda^{n} v\right\| \sim n^{m_{1}-1}\left|\lambda_{1}\right|^{n}$. The positive number $\lambda:=\left|\lambda_{1}\right|$ is the spectral radius of $\Lambda$. The integer $m:=m_{1}$ is called the multiplicity of the spectral radius.

We say that $F:=F_{1} \oplus \cdots \oplus F_{\nu}$ is the dominant eigenspace and that $F^{\prime}:=\bigoplus F_{i}$ with $1 \leq i \leq \nu, \lambda_{i}=\lambda$, is the strictly dominant eigenspace of $\Lambda$. These spaces are invariant under $\Lambda$. For any $1 \leq j \leq \nu$, there is a unique $\theta_{j} \in \mathbb{S}:=\mathbb{R} / 2 \pi \mathbb{Z}$ such that $\lambda_{j}=\lambda \exp \left(i \theta_{j}\right)$. We say that $\theta:=\left(\theta_{1}, \ldots, \theta_{\nu}\right) \in \mathbb{S}^{\nu}$ is the dominant direction of $\Lambda$; the dominant direction of $\Lambda^{n}$ is equal to $n \theta$. Denote by $\Theta$ the closed subgroup of $\mathbb{S}^{\nu}$ generated by $\theta$. It is a finite union of real tori. The orbit of each point $\theta^{\prime} \in \Theta$ under the translation $\theta^{\prime} \mapsto \theta^{\prime}+\theta$ is dense in $\Theta$. If $\lambda_{i}=\lambda$ for every $1 \leq i \leq \nu$, we have $F=F^{\prime}, \theta=0$ and $\Theta=\{0\}$.

For every linear map $L=\left(L_{1}, \ldots, L_{r}\right)$ in

$$
\operatorname{End}(E, E)=\operatorname{End}\left(E, E_{1}\right) \oplus \cdots \oplus \operatorname{End}\left(E, E_{r}\right)
$$

let

$$
\exp (-i n \theta) L:=\left(\exp \left(-i n \theta_{1}\right) L_{1}, \ldots, \exp \left(-i n \theta_{\nu}\right) L_{\nu}, L_{\nu+1}, \ldots, L_{r}\right) .
$$

We also define

$$
\Lambda_{n}:=\frac{\Lambda^{n}}{n^{m-1} \lambda^{n}} \text { and } \Lambda_{N}^{\prime}:=\frac{1}{N} \sum_{n=1}^{N} \Lambda_{i} .
$$

Let $\pi: F \longrightarrow F^{\prime}$ denote the canonical projection. The proof of the following proposition is left to the reader. We can deduce it from the proof of Proposition 2.4 if we take $K=E$ and $u=g=$ id.

Proposition 2.3. There exists a unique linear map $\Lambda_{\infty}: E \longrightarrow F$ such that

$$
\left\|\exp (-i n \theta) \Lambda_{n}-\Lambda_{\infty}\right\|=\mathrm{O}(1 / n) \text { and }\left\|\Lambda_{N}^{\prime}-\pi \circ \Lambda_{\infty}\right\|=\mathrm{O}(\log N / N) .
$$

Moreover, $\pi \circ \Lambda_{\infty}$ is of rank $\operatorname{dim} F^{\prime}$. If $\Lambda$ preserves a convex cone $\mathcal{K}$ which generates $E$ and satisfies $-\overline{\mathcal{K}} \cap \overline{\mathcal{K}}=\{0\}$, then $\lambda$ is a dominant eigenvalue of $\Lambda$ with an eigenvector in $\overline{\mathcal{K}}$.

The last property of Proposition 2.3 is the Perron-Frobenius Theorem.

Proposition 2.4. Let $K$ be a compact metric space, and let $u: K \longrightarrow E$ be a $\nu$-Hölder continuous vector-valued function. Let $g: K \longrightarrow K$ be a Lipschitz map such that $|g(x)-g(y)| \leq M|x-y|$ where $M>1$ is a constant. Assume that the spectral radius $\lambda$ of $\Lambda$ is strictly larger than 1 . Define

$$
v_{n}:=\frac{1}{n^{m-1} \lambda^{n}} \sum_{j=1}^{n} \Lambda^{j} \circ u \circ g^{n-j} \text { and } w_{N}:=\frac{1}{N} \sum_{n=1}^{N} v_{n} .
$$

Then there exist vector-valued functions $v$ and $w$ on $K$ such that

$$
\left\|\exp (-i n \theta) v_{n}-v\right\|_{\infty}=\mathrm{O}(1 / n) \text { and }\left\|w_{N}-w\right\|_{\infty}=\mathrm{O}(\log N / N) .
$$

Moreover, $v$ and $w$ are $\nu^{\prime}$-Hölder continuous for every $\nu^{\prime}>0$ with $\nu^{\prime} \leq \nu$ and $\nu^{\prime}<\log \lambda / \log M$. 
Proof. We can assume that the matrix $A$ of $\Lambda$ is the Jordan matrix $J_{\eta, m}$ with $\eta=\exp (i \theta) \lambda$ and $\theta \in \mathbb{R} / 2 \pi \mathbb{Z}$. Every entry of $A^{n}$ is of order at most $n^{m-2} \lambda^{n}$ except the one of index $(1, m)$ which is of order $n^{m-1} \lambda^{n}$. Since the functions $u \circ g^{n}$ are uniformly bounded, every entry of $A^{n}$, whose order is smaller than or equal to $n^{m-2} \lambda^{n}$, does not contribute to $\lim v_{n}$ or $\lim w_{N}$. This follows from the estimate $\sum_{j=0}^{n} j^{m-2} \lambda^{j} \lesssim n^{m-2} \lambda^{n}$ for $\lambda>1$. Hence we can suppose that all the coordinate functions of $u$ are zero except the last one.

Let $u^{+}$be the last coordinate function of $u$. Define $u_{j}^{+}:=u^{+} \circ g^{j}$ for $j \geq 0$ and $s_{j}:=\left(\begin{array}{c}j \\ m-1\end{array}\right) \eta^{j-m+1}$ for $j \geq m-1$ with the convention that $\left(\begin{array}{l}0 \\ 0\end{array}\right)=0$. The first coordinate functions of $v_{n}$ and $w_{n}$ are

$$
v_{n}^{+}:=\frac{1}{n^{m-1} \lambda^{n}} \sum_{j=m-1}^{n} s_{j} u_{n-j}^{+} \quad \text { and } \quad w_{N}^{+}:=\frac{1}{N} \sum_{n=1}^{N} v_{n}^{+} .
$$

It is sufficient to study the sequences of functions $\left(v_{n}^{+}\right)$and $\left(w_{N}^{+}\right)$.

We show first that $\left\|v_{n+1}^{+}-\exp (i \theta) v_{n}^{+}\right\|_{\infty} \lesssim n^{-2}$. Observe that $\left\|u_{j}^{+}\right\|_{\infty} \leq\left\|u^{+}\right\|_{\infty}$ and

$$
S_{j, n}:=\left|\frac{s_{j+1}}{(n+1)^{m-1} \lambda^{n+1}}-\frac{\eta s_{j}}{n^{m-1} \lambda^{n+1}}\right| \lesssim \frac{n-j+1}{n^{2} \lambda^{n-j}}
$$

Hence

$$
\begin{aligned}
& \left|v_{n+1}^{+}-\exp (i \theta) v_{n}^{+}\right| \\
& \quad=\left|\frac{1}{(n+1)^{m-1} \lambda^{n+1}} \sum_{j=m-2}^{n} s_{j+1} u_{n-j}^{+}-\frac{1}{n^{m-1} \lambda^{n+1}} \sum_{j=m-1}^{n} \eta s_{j} u_{n-j}^{+}\right| \\
& \quad \leq \frac{\left|s_{m-1}\right|\left\|u_{n-m+2}^{+}\right\|_{\infty}}{(n+1)^{m-1} \lambda^{n+1}}+\sum_{j=m-1}^{n} S_{j, n}\left\|u_{n-j}^{+}\right\|_{\infty} \lesssim n^{-2} .
\end{aligned}
$$

Thus, the sequence $\exp (-i n \theta) v_{n}^{+}$converges uniformly to a function $v^{+}$and $\left\|\exp (-i n \theta) v_{n}^{+}-v^{+}\right\|_{\infty}=\mathrm{O}(1 / n)$. It follows that $\left\|w_{N}^{+}-w^{+}\right\|_{\infty}=\mathrm{O}(\log N / N)$ with $w^{+}=0$ if $\theta \neq 0$ and $w^{+}=v^{+}$if $\theta=0$.

We only have to prove that $v^{+}$is Hölder continuous. We will use the additive notation for the distance on $K$. Let $x, y \in K$ and $\delta:=|x-y|$. First, consider the case where $\nu=\log \lambda / \log M$, so $\lambda=M^{\nu}$. Since $u^{+}$is $\nu$-Hölder continuous, there exists $c>0$, independent of $x, y$ such that

$$
\left|u^{+} \circ g^{j}(x)-u^{+} \circ g^{j}(y)\right| \leq c\left|g^{j}(x)-g^{j}(y)\right|^{\nu} .
$$

Hence

$$
\left|u^{+} \circ g^{j}(x)-u^{+} \circ g^{j}(y)\right| \leq c M^{j \nu} \delta^{\nu} .
$$


We also have $\left|u^{+} \circ g^{j}(x)-u^{+} \circ g^{j}(y)\right| \leq 2\left\|u^{+}\right\|_{\infty}$. Let $q$ be the integer part of $-\log \delta / \log M$. We have the following estimates:

$$
\begin{aligned}
\left|v_{n}^{+}(x)-v_{n}^{+}(y)\right| & \leq \frac{1}{n^{m-1} \lambda^{n}} \sum_{j=m-1}^{n} s_{j}\left|u^{+} \circ g^{n-j}(x)-u^{+} \circ g^{n-j}(y)\right| \\
& \lesssim \frac{1}{n^{m-1} \lambda^{n}} \sum_{j=m-1}^{n} j^{m-1} \lambda^{j}\left|u^{+} \circ g^{n-j}(x)-u^{+} \circ g^{n-j}(y)\right| \\
& \lesssim \sum_{j=0}^{\infty} \lambda^{-j}\left|u^{+} \circ g^{j}(x)-u^{+} \circ g^{j}(y)\right| \\
& \lesssim \sum_{j=0}^{q} \lambda^{-j} M^{j \nu} \delta^{\nu}+\sum_{j=q+1}^{\infty} \lambda^{-j} \\
& \lesssim q \delta^{\nu}+\lambda^{-q} \lesssim-(\log \delta) \delta^{\nu} .
\end{aligned}
$$

Consequently, $\left|v_{n}^{+}(x)-v_{n}^{+}(y)\right| \lesssim \delta^{\nu^{\prime}}$. In the limit, we get $\left|v^{+}(x)-v^{+}(y)\right| \lesssim|x-y|^{\nu^{\prime}}$. This is the required inequality.

The case where $\nu>\log \lambda / \log M$ is derived from the case $\nu=\log \lambda / \log M$. If $\nu<\log \lambda / \log M$, we have $M^{\nu}<\lambda$. In the same way, we obtain

$$
\left|v_{n}^{+}(x)-v_{n}^{+}(y)\right| \lesssim \sum_{j=0}^{\infty} \lambda^{-j} M^{j \nu} \delta^{\nu} \lesssim \delta^{\nu} .
$$

2.3. Action on cohomology groups. By Hodge theory [25, 9, we have the decomposition

$$
\mathcal{H}^{n}(X, \mathbb{C})=\sum_{p+q=n} \mathcal{H}^{p, q}(X, \mathbb{C})
$$

where $\mathcal{H}^{p, q}(X, \mathbb{C})$ is the subspace of $\mathcal{H}^{n}(X, \mathbb{C})$ spanned by classes of closed $(p, q)$ forms. The group $\mathcal{H}^{p, q}(X, \mathbb{C})$ is isomorphic to the Dolbeault cohomology group of bidegree $(p, q)$. We also have $\mathcal{H}^{p, q}(X, \mathbb{C})=\overline{\mathcal{H}^{q, p}(X, \mathbb{C})}$. Define

$$
\mathcal{H}^{p, p}(X, \mathbb{R}):=\mathcal{H}^{p, p}(X, \mathbb{C}) \cap \mathcal{H}^{2 p}(X, \mathbb{R}) .
$$

Then

$$
\mathcal{H}^{p, p}(X, \mathbb{C})=\mathcal{H}^{p, p}(X, \mathbb{R}) \otimes_{\mathbb{R}} \mathbb{C} .
$$

The Kähler cone $\mathcal{K}_{X}$ of $\mathcal{H}^{1,1}(X, \mathbb{R})$ is the cone of classes of Kähler forms on $X$. In what follows, $\mathcal{K}_{X}^{*}$ will denote the cone of classes of positive closed $(1,1)$-currents. These cones are convex; $\mathcal{K}_{X}$ is open; $\mathcal{K}_{X}^{*}$ is closed. We also have $\overline{\mathcal{K}}_{X} \subset \mathcal{K}_{X}^{*}$ and $\left(-\mathcal{K}_{X}^{*}\right) \cap \mathcal{K}_{X}^{*}=\{0\}$.

We will construct some invariant currents by induction starting from an invariant current. It is necessary to introduce cohomology groups relative to a positive closed current: the manifold $X$ is replaced by a current $T$. Let $T \neq 0$ be a positive closed current of bidegree $(s, s)$ with $0 \leq s \leq k-1$. For any $1 \leq p \leq k-s$, let $N^{p, p}(T, \mathbb{R})$ denote the space of classes $[\alpha] \in \mathcal{H}^{p, p}(X, \mathbb{R})$ which satisfy

$$
[T] \wedge[\alpha]=0 \text { in } \mathcal{H}^{p+s, p+s}(X, \mathbb{R}) .
$$

Let $N_{\nu}^{1,1}(T, \mathbb{R})$ denote the space of classes $[S]$ where $S$ is a closed $(1,1)$-current (not necessarily positive) with a $\nu$-Hölder continuous potential such that $T \wedge S=0$. 
Define

$$
\mathcal{H}^{p, p}(T, \mathbb{R}):=\frac{\mathcal{H}^{p, p}(X, \mathbb{R})}{N^{p, p}(T, \mathbb{R})} \text { and } \mathcal{H}_{\nu}^{1,1}(T, \mathbb{R}):=\frac{\mathcal{H}^{1,1}(X, \mathbb{R})}{N_{\nu}^{1,1}(T, \mathbb{R})}
$$

The map $\pi_{T}: \mathcal{H}^{p, p}(T, \mathbb{R}) \longrightarrow \mathcal{H}^{p+s, p+s}(X, \mathbb{R})$, given by $[\alpha] \mapsto[T] \wedge[\alpha]$, is injective. Hence $\|[T] \wedge$.$\| is a norm on \mathcal{H}^{p, p}(T, \mathbb{R})$ if $\|$.$\| is a norm on \mathcal{H}^{p+s, p+s}(X, \mathbb{R})$.

Let $f$ be a holomorphic automorphism of $X$ such that $f^{*} T=\lambda_{T} T$ where $\lambda_{T}>0$ is a constant. We define the map $f^{*}$ on $\mathcal{H}^{p, p}(X, \mathbb{R})$ and $\mathcal{H}^{p, p}(T, \mathbb{R})$ by $f^{*}[\alpha]=$ $\left[f^{*}(\alpha)\right]$ for every closed $(p, p)$-current $\alpha$ on $X$. Let

$$
\lambda_{p, n}(T):=\lambda_{T}^{-n}\left\|\left[\left(f^{n}\right)^{*}\left(T \wedge \omega^{p}\right)\right]\right\|=\left\|\left[T \wedge\left(f^{n}\right)^{*} \omega^{p}\right]\right\|
$$

and

$$
\lambda_{p}(T):=\limsup _{n \rightarrow \infty} \lambda_{p, n}(T)^{1 / n}=\limsup _{n \rightarrow \infty}\left(\int_{X} T \wedge\left(f^{n}\right)^{*} \omega^{p} \wedge \omega^{k-s-p}\right)^{1 / n} .
$$

Observe that $\lambda_{p}(T)$ depends neither on the Kähler form $\omega$ nor on the norm $\|$.$\| on$ $\mathcal{H}^{p+s, p+s}(X, \mathbb{R})$. The following proposition follows from the discussion on Jordan forms for linear maps and from Proposition 2.3 (see also [17, 18]).

Proposition 2.5. Let $X, f$ and $T$ be as above. Then, $\lambda_{p}(T)$ is the spectral radius of $f^{*}$ on $\mathcal{H}^{p, p}(T, \mathbb{R})$. If $l_{p}(T)$ is its multiplicity, then

$$
\lambda_{p, n}(T) \sim n^{l_{p}(T)-1} \lambda_{p}(T)^{n} .
$$

In particular, $\lambda_{p, n}(T)^{1 / n}$ converge to $\lambda_{p}(T)$.

Proposition 2.6. Let $X, T, f$ be as above. Then for every $p_{1} \geq 1$ and $p_{2} \geq 1$ such that $p_{1}+p_{2} \leq k-s$, we have $\lambda_{p_{1}+p_{2}}(T) \leq \lambda_{p_{1}}(T) \lambda_{p_{2}}(T)$. In particular, $\lambda_{1}(T)^{p} \geq \lambda_{p}(T)$ for $1 \leq p \leq k-s$ and $\lambda_{1}(T)^{k-s} \geq \lambda_{T}^{-1}$.

Proof. Propositions 2.3 and 2.5 imply the existence of $\left[\alpha_{1}\right] \in \mathcal{H}^{p_{1}, p_{1}}(T, \mathbb{R}),\left[\alpha_{2}\right] \in$ $\mathcal{H}^{p_{2}, p_{2}}(T, \mathbb{R})$ such that

$$
\frac{\left[\left(f^{n}\right)^{*} \omega^{p_{1}}\right]}{n^{l_{p_{1}}(T)-1} \lambda_{p_{1}}(T)^{n}} \longrightarrow\left[\alpha_{1}\right] \text { and } \frac{\left[\left(f^{n}\right)^{*} \omega^{p_{2}}\right]}{n^{l_{p_{2}}(T)-1} \lambda_{p_{2}}(T)^{n}} \longrightarrow\left[\alpha_{2}\right] .
$$

Hence

$$
\frac{\left[\left(f^{n}\right)^{*} \omega^{p_{1}+p_{2}}\right]}{n^{l_{p_{1}}(T)+l_{p_{2}}(T)-2} \lambda_{p_{1}}(T)^{n} \lambda_{p_{2}}(T)^{n}} \longrightarrow\left[\alpha_{1}\right] \wedge\left[\alpha_{2}\right]
$$

in $\mathcal{H}^{p_{1}+p_{2}, p_{1}+p_{2}}(T, \mathbb{R})$. On the other hand, there exists a non-zero class $[\alpha] \in$ $\mathcal{H}^{p_{1}+p_{2}, p_{1}+p_{2}}(T, \mathbb{R})$ such that

$$
\frac{\left[\left(f^{n}\right)^{*} \omega^{p_{1}+p_{2}}\right]}{n^{l_{p_{1}+p_{2}}(T)-1} \lambda_{p_{1}+p_{2}}(T)^{n}} \longrightarrow[\alpha] .
$$

The property $[\alpha] \neq 0$ implies that $\lambda_{p_{1}+p_{2}}(T) \leq \lambda_{p_{1}}(T) \lambda_{p_{2}}(T)$.

The inequality $\lambda_{1}(T)^{p} \geq \lambda_{p}(T)$ is clear. Since $f$ is an automorphism, the mass of the measure $\left(f^{n}\right)^{*}\left(T \wedge \omega^{k-s}\right)$ is equal to the mass of $T \wedge \omega^{k-s}$. Thus, $\lambda_{T} \lambda_{k-s}(T)=1$ and $\lambda_{1}(T)^{k-s} \geq \lambda_{k-s}(T)=\lambda_{T}^{-1}$.

The space $\mathcal{H}_{\nu}^{1,1}(T, \mathbb{R})$ is invariant under $f^{*}$. Let $\rho_{\nu}(T)$ denote the spectral radius of $f^{*}$ on $\mathcal{H}_{\nu}^{1,1}(T, \mathbb{R})$ and let $m_{\nu}(T)$ denote its multiplicity. Since $N_{\nu}^{1,1}(T, \mathbb{R}) \subset$ $N^{1,1}(T, \mathbb{R})$, we have

$$
\left(\lambda_{1}(T), l_{1}(T)\right) \leq\left(\rho_{\nu}(T), m_{\nu}(T)\right)
$$


We will prove later (see Lemma 3.3) that if $\lambda_{1}(T)>1$, and if $\nu$ is small enough, then the last inequality is in fact an equality.

When $T$ is the integration current on $X$, we define

$$
d_{p, n}:=\left\|\left[\left(f^{n}\right)^{*} \omega^{p}\right]\right\| \text { and } d_{p}:=\lim _{n \rightarrow \infty} \sqrt[n]{d_{p, n}}
$$

We also have

$$
d_{p}=\lim _{n \rightarrow \infty}\left(\int_{X}\left(f^{n}\right)^{*} \omega^{p} \wedge \omega^{k-p}\right)^{1 / n} .
$$

The numbers $d_{p}$ are called the dynamical degrees of $f$. The last one $d_{t}:=d_{k}$ is the topological degree of $f$ which is equal to 1 because $f$ is an automorphism. It is noted in [29] that an inequality of Khovanskii-Teissier-Gromov shows that $p \mapsto \log d_{p}$ is concave; hence the sequence $\left(d_{p-1} / d_{p}\right)_{1 \leq p \leq k}$ is increasing [31, 36, 27]. In particular, there exist $m, m^{\prime}, 1 \leq m \leq m^{\prime} \leq k-1$, such that

$$
1 \leq d_{1}<\cdots<d_{m}=\cdots=d_{m^{\prime}}>\cdots>d_{k}=1 .
$$

From Proposition 2.5, we know that $d_{p}$ is the spectral radius of $f^{*}$ acting on $\mathcal{H}^{p, p}(X, \mathbb{R})$. Let $l_{p}$ denote its multiplicity. Since $f^{*}$ preserves the cone of classes of positive closed $(p, p)$-currents, by Proposition $2.3, d_{p}$ is a dominant eigenvalue of $f^{*}$ on $\mathcal{H}^{p, p}(X, \mathbb{R})$. The relation

$$
\int_{X}\left(f^{n}\right)^{*} \omega^{p} \wedge \omega^{k-p}=\int_{X} \omega^{p} \wedge\left(f^{n}\right)_{*} \omega^{k-p}
$$

implies that the spectral radius of $f_{*}$ on $\mathcal{H}^{k-p, k-p}(X, \mathbb{R})$ is also equal to $d_{p}$ and its multiplicity is equal to $l_{p}$.

According to the Gromov-Yomdin theorem, the topological entropy $\mathrm{h}(f)$ of $f$ is equal to $\max _{1 \leq p \leq k} \log d_{p}$ [26, 38, 17, 18. In particular, if $\mathrm{h}(f)>0$, we have $\max d_{p}>1$. It follows from Proposition 2.6 that $d_{1}>1$. It is shown in 15 that the map which associates to a holomorphic endomorphism of $X$ its topological entropy has discrete image in $[0,+\infty[$. It is well known that if $f$ belongs to the component of the identity in the automorphism group of $X$, then $\mathrm{h}(f)=0$.

\section{Relative Green currents}

Let $f$ be a holomorphic automorphism of a compact Kähler manifold $(X, \omega)$ of dimension $k$. Let $T$ be a positive closed $(s, s)$-current, $0 \leq s \leq k-1$, on $X$, which satisfies a relation $f^{*} T=\lambda_{T} T, \lambda_{T}>0$. When $s=0, T$ is a multiple of the integration current on the manifold $X$ and $\lambda_{T}=1$. Define

$$
M_{n}:=\left\|\mathrm{D} f^{n}\right\|_{\infty} \text { and } M:=\lim _{n \rightarrow \infty} M_{n}^{1 / n}
$$

where $\mathrm{D} f^{n}$ is the differential of $f^{n}$. The constant $M$ is independent of the metric on $X$.

Assume that $\lambda_{1}(T)>1$. Let $\Gamma(T)$ denote the cone of $(s+1, s+1)$-currents $T \wedge S$ where $S$ is a closed $(1,1)$-current (not necessarily positive) satisfying the following properties:

(1) $S$ has a $\nu$-Hölder continuous potential for every $\nu$ such that $0<\nu<$ $\log \lambda_{1}(T) / \log M$

(2) $T \wedge S$ is a positive current;

(3) $T \wedge f^{*} S=\lambda_{1}(T) T \wedge S$. 
We will denote by $\mathbb{R} \Gamma(T)$ the real space generated by $\Gamma(T)$. We can now describe $\Gamma(T)$.

Theorem 3.1. Let $X, T$ and $f$ be as above. Assume that $\lambda_{1}(T)>1$. Then, $\Gamma(T)$ is a closed finite dimensional cone with non-zero elements. Let $R$ be a closed real $(1,1)$-current with a continuous potential. Then, the sequence of currents

$$
\frac{1}{N} \sum_{n=1}^{N} \frac{T \wedge\left(f^{n}\right)^{*} R}{n^{l_{1}(T)-1} \lambda_{1}(T)^{n}}
$$

converges to a current in $\mathbb{R} \Gamma(T)$, which depends only on the class $[R]$ in $\mathcal{H}^{1,1}(X, \mathbb{R})$. If $[R]$ belongs to $\mathcal{K}_{X}$, the limit current belongs to $\Gamma(T) \backslash\{0\}$.

Fix $\nu$ such that $0<\nu<\log \lambda_{1}(T) / \log M$. Replacing $f$ by $f^{n}$ with $n>>0$, we can assume that $0<\nu<\log \lambda_{1}(T) / \log M_{1}$. Let $m$ be the largest integer such that $[\omega],\left[f^{*} \omega\right], \ldots,\left[\left(f^{m-1}\right)^{*} \omega\right]$ are linearly independent in $\mathcal{H}_{\nu}^{1,1}(T, \mathbb{R})$. Then there exist real numbers $a_{0}, \ldots, a_{m-1}$ such that we have in $\mathcal{H}_{\nu}^{1,1}(T, \mathbb{R})$

$$
\left[\left(f^{m}\right)^{*} \omega\right]=a_{m-1}\left[\left(f^{m-1}\right)^{*} \omega\right]+\cdots+a_{0}[\omega] .
$$

Let $E$ be the subspace of $\mathcal{H}_{\nu}^{1,1}(T, \mathbb{R})$ generated by $[\omega], \ldots,\left(f^{m-1}\right)^{*}[\omega]$. These $m$ classes form a basis $\mathcal{B}$ of $E$ and $E$ is invariant under $f^{*}$. Denote by $\Lambda$ the restriction of $f^{*}$ to $E$. The notation $\theta, \theta^{\prime}, \Theta, \rho_{\nu}(T), m_{\nu}(T)$ was introduced in Section 2.

The matrix of $\Lambda$ with respect to $\mathcal{B}$ is

$$
A:=\left(\begin{array}{ccccc}
0 & 0 & \cdots & 0 & a_{0} \\
1 & 0 & \cdots & 0 & a_{1} \\
0 & 1 & \cdots & 0 & a_{2} \\
\vdots & \vdots & \ddots & \vdots & \vdots \\
0 & 0 & \cdots & 1 & a_{m-1}
\end{array}\right) .
$$

Since $E$ contains a Kähler class, the spectral radii of $\Lambda$ and of $A$ are equal to $\rho_{\nu}(T) \geq \lambda_{1}(T)>1$. Moreover, their multiplicities are equal to $m_{\nu}(T)$. We have

$$
\left\|\Lambda^{n}\right\|=\left\|A^{n}\right\| \sim n^{m_{\nu}(T)-1} \rho_{\nu}(T)^{n} .
$$

Lemma 3.2. There exist a closed $(1,1)$-current $S$ and a continuous family of closed $(1,1)$-currents $S_{\theta^{\prime}}$ with $\nu$-Hölder continuous potentials, for $\theta^{\prime} \in \Theta$, such that $T \wedge S \neq$ $0, T \wedge S_{\theta^{\prime}} \neq 0, T \wedge f^{*} S=\rho_{\nu}(T) T \wedge S$ and $T \wedge f^{*} S_{\theta^{\prime}}=\rho_{\nu}(T) T \wedge S_{\theta^{\prime}+\theta}$. Moreover, the sequences of positive closed currents

$$
\widetilde{Z}_{N}:=\frac{1}{N} \sum_{n=1}^{N} \frac{T \wedge\left(f^{n}\right)^{*} \omega}{n^{m_{\nu}(T)-1} \rho_{\nu}(T)^{n}} \quad \text { and } \quad Z_{n_{i}}:=\frac{T \wedge\left(f^{n_{i}}\right)^{*} \omega}{n_{i}^{m_{\nu}(T)-1} \rho_{\nu}(T)^{n_{i}}}
$$

converge to $T \wedge S$ and to $T \wedge S_{\theta^{\prime}}$ when $N \rightarrow \infty$ and $n_{i} \rightarrow \infty$ with, respectively, $n_{i} \theta \rightarrow \theta^{\prime}$. In particular, if $\Theta$ is reduced to one point, $Z_{n}$ converge to $T \wedge S$.

Proof. From the definition of $\mathcal{H}_{\nu}^{1,1}(T, \mathbb{R})$, there exists a $(1,1)$-current $R$ with a $\nu$-Hölder continuous potential such that $T \wedge R=0$ and

$$
\left[\left(f^{m}\right)^{*} \omega-\sum_{j=1}^{m} a_{m-j}\left(f^{m-j}\right)^{*} \omega\right]=[R] \text { in } \mathcal{H}^{1,1}(X, \mathbb{R}) .
$$


Hence, there exists a $\nu$-Hölder continuous function $u$ such that

$$
\left(f^{m}\right)^{*} \omega-\sum_{j=1}^{m} a_{m-j}\left(f^{m-j}\right)^{*} \omega=R+\mathrm{dd}^{\mathrm{c}} u .
$$

Then

$$
T \wedge\left(\left(f^{m}\right)^{*} \omega-\sum_{j=1}^{m} a_{m-j}\left(f^{m-j}\right)^{*} \omega\right)=T \wedge \mathrm{dd}^{\mathrm{c}} u .
$$

Define

$$
W_{n}:=\left(\begin{array}{c}
\left(f^{n}\right)^{*} \omega \\
\vdots \\
\left(f^{n+m-2}\right)^{*} \omega \\
\left(f^{n+m-1}\right)^{*} \omega
\end{array}\right) \quad \text { and } \quad U:=\left(\begin{array}{c}
0 \\
\vdots \\
0 \\
u
\end{array}\right) .
$$

Then $W_{n+1}=f^{*} W_{n}$ and $T \wedge W_{1}=T \wedge B W_{0}+T \wedge \mathrm{dd}^{\mathrm{c}} U$ where $B$ is the transpose of $A$. By induction, we obtain

$$
T \wedge W_{n}=T \wedge\left(B^{n} W_{0}+\mathrm{dd}^{\mathrm{c}} \sum_{j=1}^{n} B^{j-1} U \circ f^{n-j}\right) .
$$

Define

$$
\bar{W}_{n}:=\frac{W_{n}}{n^{m_{\nu}(T)-1} \rho_{\nu}(T)^{n}}
$$

and

$$
V_{n}:=\frac{1}{n^{m_{\nu}(T)-1} \rho_{\nu}(T)^{n}}\left(B^{n} W_{0}+\mathrm{dd}^{\mathrm{c}} \sum_{j=1}^{n} B^{j-1} U \circ f^{n-j}\right) .
$$

Denote by $\bar{W}_{n}^{+}$and $V_{n}^{+}$the first components of $\bar{W}_{n}$ and of $V_{n}$. We have $\left[\bar{W}_{n}^{+}\right]=$ $\left[V_{n}^{+}\right]$in $\mathcal{H}_{\nu}^{1,1}(T, \mathbb{R})$. Proposition 2.3 implies that the sequence of classes $\left[\bar{W}_{n}^{+}\right]$is bounded. Moreover, since $\omega$ is a Kähler form, any cluster point of this sequence is a non-zero class. Indeed, every class is bounded by $C[\omega]$.

Propositions 2.3 and 2.4 imply that when $\left(n_{i} \theta\right)$ converges to $\theta^{\prime}$, the sequence $V_{n_{i}}^{+}$converges to a current $S_{\theta^{\prime}}$ with a $\nu$-Hölder continuous potential. Moreover, $S_{\theta^{\prime}}$ depends on $\theta^{\prime}$ but not on $\left(n_{i}\right)$. The current $T \wedge S_{\theta^{\prime}}$ is positive and closed. Since $\left[S_{\theta^{\prime}}\right] \neq 0$ in $\mathcal{H}_{\nu}^{1,1}(T, \mathbb{R})$, from the definition of $\mathcal{H}_{\nu}^{1,1}(T, \mathbb{R})$, we have $T \wedge S_{\theta^{\prime}} \neq 0$. It is clear that $S_{\theta^{\prime}}$ depends continuously on $\theta^{\prime} \in \Theta$. Since $\lim \left(n_{i}+1\right) \theta=\theta^{\prime}+\theta$, we have

$$
\begin{aligned}
T \wedge f^{*} S_{\theta^{\prime}}-\rho_{\nu}(T) T \wedge S_{\theta^{\prime}+\theta} & =\lim \left(\frac{1}{\lambda_{T}} f^{*} Z_{n_{i}}-\rho_{\nu}(T) Z_{n_{i}+1}\right) \\
& =\rho_{\nu}(T) \lim \left(\left[\frac{n_{i}+1}{n_{i}}\right]^{m_{\nu}(T)-1} Z_{n_{i}+1}-Z_{n_{i}+1}\right) \\
& =0 .
\end{aligned}
$$

Hence, $T \wedge f^{*} S_{\theta^{\prime}}=\rho_{\nu}(T) T \wedge S_{\theta^{\prime}+\theta}$.

Propositions 2.3 and 2.4 imply that the sequence of currents $\frac{1}{N} \sum_{n=1}^{N} V_{n}^{+}$converges to a current $S$ which has a $\nu$-Hölder continuous potential. We also have $T \wedge S \neq 0$ because every limit value of $\left(T \wedge V_{n}^{+}\right)$is a non-zero positive current. In the same way, we get $T \wedge f^{*} S=\rho_{\nu}(T) T \wedge S$. 
Lemma 3.3. Under the hypothesis of Theorem 3.1, we have

$$
\left(\rho_{\nu}(T), m_{\nu}(T)\right)=\left(\lambda_{1}(T), l_{1}(T)\right)
$$

for every $\nu$ such that $0<\nu<\log \lambda_{1}(T) / \log M$.

Proof. Recall that the map $\pi: \mathcal{H}^{1,1}(T, \mathbb{R}) \longrightarrow \mathcal{H}^{s+1, s+1}(X, \mathbb{R})$ defined by $\pi([\alpha]):=$ $[T] \wedge[\alpha]$ is injective. Consequently, the spectral radius of $f^{*}$ on $\pi\left(\mathcal{H}^{1,1}(T, \mathbb{R})\right)$ is equal to $\lambda_{T} \lambda_{1}(T)$ and its multiplicity is equal to $l_{1}(T)$. We have seen that the sequence of classes

$$
\frac{\left[\left(f^{n_{i}}\right)^{*}(T \wedge \omega)\right]}{n_{i}^{m_{\nu}(T)-1} \lambda_{T}^{n_{i}} \rho_{\nu}(T)^{n_{i}}}
$$

converges to $\left[T \wedge S_{\theta^{\prime}}\right]$ in $\mathcal{H}^{s+1, s+1}(X, \mathbb{R}) \backslash\{0\}$. It follows from Proposition 2.5 that $\left(\rho_{\nu}(T), m_{\nu}(T)\right)=\left(\lambda_{1}(T), l_{1}(T)\right)$.

Lemma 3.4. With the assumptions of Theorem 3.1, let $R$ be a closed $(1,1)$-current with a continuous potential such that $[R]=\lambda[\omega]$ in $\mathcal{H}^{1,1}(X, \mathbb{R})$ with $\lambda \in \mathbb{R}$. Then the sequence of currents

$$
\frac{1}{N} \sum_{n=1}^{N} \frac{T \wedge\left(f^{n}\right)^{*} R}{n^{l_{1}(T)-1} \lambda_{1}(T)^{n}}
$$

converges to $\lambda T \wedge S$. In particular, the limit is 0 if $[R]=0$ in $\mathcal{H}^{1,1}(X, \mathbb{R})$.

Proof. Let $u$ be a continuous function such that $R=\lambda \omega+\mathrm{dd}^{\mathrm{c}} u$. We have

$$
\frac{T \wedge\left(f^{n}\right)^{*} R}{n^{l_{1}(T)-1} \lambda_{1}(T)^{n}}=\lambda \frac{T \wedge\left(f^{n}\right)^{*} \omega}{n^{l_{1}(T)-1} \lambda_{1}(T)^{n}}+T \wedge \mathrm{dd}^{\mathrm{c}}\left(\frac{u \circ f^{n}}{n^{l_{1}(T)-1} \lambda_{1}(T)^{n}}\right) .
$$

Since the $u \circ f^{n}$ are uniformly bounded, the last relation implies that the sequence of currents

$$
\frac{T \wedge\left(f^{n}\right)^{*} R}{n^{l_{1}(T)-1} \lambda_{1}(T)^{n}}-\lambda \frac{T \wedge\left(f^{n}\right)^{*} \omega}{n^{l_{1}(T)-1} \lambda_{1}(T)^{n}}
$$

converges to 0 . It suffices to apply Lemmas 3.2 and 3.3. The proof is valid for $R$ positive closed with a bounded potential.

End of the proof of Theorem 3.1. Let $\omega_{1}, \ldots, \omega_{j}$ be Kähler forms such that the classes $\left[\omega_{1}\right], \ldots,\left[\omega_{j}\right]$ generate $\mathcal{H}^{1,1}(X, \mathbb{R})$. We can apply Lemma 3.4 to the forms $\omega_{i}$. This implies the convergence in Theorem 3.1.

We now show that $\operatorname{dim} \Gamma(T) \leq \operatorname{dim} \mathcal{H}^{1,1}(X, \mathbb{R})$. Otherwise, there exists a nonzero current $T \wedge S$ in $\mathbb{R} \Gamma(T)$ with $[S]=0$. Let $u$ be a continuous function such that $S=\mathrm{dd}^{\mathrm{c}} u$. We have

$$
T \wedge S=\lim \frac{T \wedge\left(f^{n}\right)^{*} S}{\lambda_{1}(T)^{n}}=\lim T \wedge \mathrm{dd}^{\mathrm{c}}\left(\frac{u \circ f^{n}}{\lambda_{1}(T)^{n}}\right)=0 .
$$

This is impossible.

Let $T^{(1)}, \ldots, T^{(r)}$ be a maximal linearly independent set in $\Gamma(T)$. The cone $\Gamma(T)$ is equal to the intersection of the space generated by the $T^{(i)}$ and the cone of positive closed $(s+1, s+1)$-currents. Hence, it is closed.

When $T$ is the integration current on $X$, Propositions 2.3, 2.5, 2.6 and Theorem 3.1 imply the following result (see [6, 28] for the case of surfaces and [1, 223, 35] for polynomial automorphisms). 
Corollary 3.5. Let $(X, \omega)$ be a compact Kähler manifold of dimension $k$. Let $f$ be a holomorphic automorphism of $X$, of positive topological entropy. Then $d_{1}>1$ and there exists a positive closed $(1,1)$-current $T_{1}$ satisfying $f^{*} T_{1}=d_{1} T_{1}$. Moreover, the potential of $T_{1}$ is Hölder continuous and the class $\left[T_{1}\right]$ belongs to $\overline{\mathcal{K}}_{X}$.

Remarks 3.6. The construction of invariant currents in Theorem 3.1 is still valid if we restrict ourselves to an invariant subspace $E$ of $\mathcal{H}^{1,1}(X, \mathbb{R})$. We have to assume that the spectral radius of $f^{*}$ on the projection $E^{\prime}$ of $E$ in $\mathcal{H}^{1,1}(T, \mathbb{R})$ is strictly larger than 1 . The construction gives invariant currents with Hölder continuous potentials which are not necessarily positive; there is a non-zero current if $f_{\mid E^{\prime}}^{*}$ has a dominant real eigenvalue. In particular, every invariant $(1,1)$-current $T$ with continuous potential such that $f^{*} T=\lambda T,|\lambda|>1$, has a Hölder continuous potential. Let $\mathcal{K}_{X}^{b}$ be the cone of non-zero classes of positive closed $(1,1)$-currents with bounded potentials. If $E \cap \mathcal{K}_{X}^{b} \neq\{0\}$, we obtain a positive current with a Hölder continuous potential.

\section{Green Currents}

Let $f$ be as in Section 3. Recall that $d_{s}$ is the spectral radius of $f^{*}$ on $\mathcal{H}^{s, s}(X, \mathbb{R})$. Let $l_{s}$ denote its multiplicity. We consider $\Gamma_{s}$ the cone of PB positive closed $(s, s)$ currents $T$ such that $f^{*} T=d_{s} T$. Let $\mathbb{R} \Gamma_{s}$ denote the real space generated by $\Gamma_{s}$. For every positive closed $(s, s)$-current $T$, define

$$
\mathcal{C}(T):=\{S \text { positive closed }(s, s) \text {-current, } S \leq c T \text { for some } c>0\}
$$

and $\mathbb{R C}(T)$ the real space generated by $\mathcal{C}(T)$. Let [ ] denote the map which associates to a closed $(s, s)$-current its cohomology class in $\mathcal{H}^{s, s}(X, \mathbb{C})$.

Theorem 4.1. Assume that $d_{s}>d_{s-1}$. Let $S$ be a PB closed real $(s, s)$-current. Then, the sequence of currents

$$
S_{N}:=\frac{1}{N} \sum_{n=1}^{N} \frac{\left(f^{n}\right)^{*} S}{n^{l_{s}-1} d_{s}^{n}}
$$

converges to a current in $\mathbb{R} \Gamma_{s}$ which depends only on the class $[S]$ in $\mathcal{H}^{s, s}(X, \mathbb{R})$. Moreover, $\Gamma_{s} \neq\{0\}$ and the restriction of [ ] to $\mathbb{R} \Gamma_{s}$ is injective. Every current in $\mathbb{R} \Gamma_{s}$ is $P C$. If $T$ belongs to $\Gamma_{s}$, then the restriction of [] to $\mathbb{R C}(T)$ is injective. In particular, the cones $\Gamma_{s}$ and $\mathcal{C}(T)$ are finite dimensional and closed. If $[T]$ is extremal in the cone of classes of positive closed $(s, s)$-currents, then $T$ is extremal in the cone of positive closed $(s, s)$-currents.

Proof. Let $\Phi$ be a DSH $(k-s, k-s)$-current such that $\operatorname{dd}^{\mathrm{c}} \Phi=\Omega^{+}-\Omega^{-}$where $\Omega^{ \pm}$are positive closed $(k-s+1, k-s+1)$-currents. Then $\left[\Omega^{+}\right]=\left[\Omega^{-}\right]$and $\left\|\Omega^{+}\right\|=\left\|\Omega^{-}\right\|$. Assume that $\left\|\Omega^{+}\right\|=\left\|\Omega^{-}\right\| \leq 1$. Proposition 2.1 implies the existence of a $(k-s, k-s)$-form $\Phi_{0}=\Phi_{0}^{+}-\Phi_{0}^{-}$such that $\Phi_{0}^{ \pm} \leq 0, \operatorname{dd}^{c} \Phi_{0}=\mathrm{dd}^{\mathrm{c}} \Phi$ and $\left\|\Phi_{0}^{ \pm}\right\|_{\mathrm{DSH}} \leq A$. The current $\Psi_{0}:=\Phi-\Phi_{0}$ is dd $\mathrm{d}^{\mathrm{c}}$-closed. Define $\Omega_{n}^{ \pm}:=\left(f^{n}\right)_{*} \Omega^{ \pm}$. Recall that the spectral radius of $f_{*}$ on $\mathcal{H}^{k-s+1, k-s+1}(X, \mathbb{C})$ is equal to $d_{s-1}$ and that its multiplicity is equal to $l_{s-1}$ (see Section 2). Fix $\epsilon, 0<\epsilon<d_{s}-d_{s-1}$. We have $\left\|\Omega_{n}^{ \pm}\right\| \lesssim\left(d_{s}-\epsilon\right)^{n}$.

Proposition 2.1 implies the existence of $(k-s, k-s)$-forms $\Phi_{n}=\Phi_{n}^{+}-\Phi_{n}^{-}$such that $\operatorname{dd}^{\mathrm{c}} \Phi_{n}=\Omega_{n}^{+}-\Omega_{n}^{-}, \Phi_{n}^{ \pm} \leq 0$ and $\left\|\Phi_{n}^{ \pm}\right\|_{\mathrm{DSH}} \lesssim\left(d_{s}-\epsilon\right)^{n}$. If $S$ is $\mathrm{PB}$ and $\Phi$ is smooth or if $S$ is smooth and $\Phi$ is DSH as above, we have $\left|\left\langle S, \Phi_{n}^{ \pm}\right\rangle\right| \lesssim\left(d_{s}-\epsilon\right)^{n}$ for 
every $n \geq 0$. We define by induction the $\mathrm{dd}^{\mathrm{c}}$-closed form $\Psi_{n}$ as $\Psi_{n}:=f_{*} \Phi_{n-1}-\Phi_{n}$. They satisfy $\left\|\Psi_{n}\right\|_{\mathrm{L}^{1}} \lesssim\left(d_{s}-\epsilon\right)^{n}$ for $n \geq 1$. On the other hand, we have

$$
\left(f^{n}\right)_{*} \Phi=\left(f^{n}\right)_{*} \Psi_{0}+\left(f^{n}\right)_{*} \Phi_{0}=\left(f^{n}\right)_{*} \Psi_{0}+\left(f^{n-1}\right)_{*} \Psi_{1}+\left(f^{n-1}\right)_{*} \Phi_{1} .
$$

So by induction, we get

$$
\left(f^{n}\right)_{*} \Phi=\left(f^{n}\right)_{*} \Psi_{0}+\cdots+f_{*} \Psi_{n-1}+\Psi_{n}+\Phi_{n} .
$$

Since $X$ is Kähler, every closed form which is d-exact is dd $^{\mathrm{c}}$-exact 9 p. 41]. Hence the $\mathrm{dd}^{\mathrm{c}}$-closed form $\Psi_{n}$ defines a linear form on $\mathcal{H}^{s, s}(X, \mathbb{R})$ by $[\alpha] \mapsto \int \Psi_{n} \wedge \alpha$ for every real closed $(s, s)$-form $\alpha$. The Poincaré duality allows us to associate to $\Psi_{n}$ a unique class $c_{n}$ in $\mathcal{H}^{k-s, k-s}(X, \mathbb{R})$. For $n \geq 1$ we have

$$
\left\|c_{n}\right\| \lesssim\left\|\Psi_{n}\right\|_{\mathrm{L}^{1}} \lesssim\left(d_{s}-\epsilon\right)^{n} .
$$

Define

$$
b_{n}:=\left(f^{n}\right)_{*} c_{0}+\left(f^{n-1}\right)_{*} c_{1}+\cdots+c_{n} \text { and } B_{N}:=\frac{1}{N} \sum_{n=1}^{N} \frac{b_{n}}{n^{l_{s}-1} d_{s}^{n}} .
$$

As in the proof of the Proposition 2.4, we can check that the sequence $\left(B_{N}\right)$ converges to a class $B \in \mathcal{H}^{k-s, k-s}(X, \mathbb{C})$ such that $\|B\| \leq c\|\Phi\|_{\text {DSH }}$ where $c>0$ is a constant.

Now, assume that $S$ is smooth and $\Phi$ is DSH. Since $S$ is closed,

$$
\left\langle\left(f^{n}\right)^{*} S, \Phi\right\rangle=\left\langle S,\left(f^{n}\right)_{*} \Phi\right\rangle=\int[S] \wedge b_{n}+\left\langle S, \Phi_{n}\right\rangle
$$

and

$$
\left\langle S_{N}, \Phi\right\rangle=\int[S] \wedge B_{N}+\frac{1}{N} \sum_{n=1}^{N} \frac{\left\langle S, \Phi_{n}\right\rangle}{n^{l_{s}-1} d_{s}^{n}} .
$$

The second term in the right-hand side of the last equality tends to zero because $\left\|\Phi_{n}\right\|_{\mathrm{DSH}} \lesssim\left(d_{s}-\epsilon\right)^{n}$. Hence

$$
\lim \left\langle S_{N}, \Phi\right\rangle=\int[S] \wedge B \leq c\|\Phi\|_{\mathrm{DSH}} .
$$

It follows that $\left(S_{N}\right)$ converges to a $\mathrm{PB}$ current $S_{\infty}$ which depends only on the class $[S]$. It is clear that $f^{*} S_{\infty}=d_{s} S_{\infty}$. Hence $S_{\infty}$ belongs to $\mathbb{R} \Gamma_{s}$ (we can write $S$ and $S_{\infty}$ as differences of positive closed currents). Observe that if $S$ is strictly positive, by definition of $d_{s}$ and $l_{s}$, we have $\left[S_{\infty}\right] \neq 0$. Hence $S_{\infty}$ is a non-zero positive current and $\Gamma_{s} \neq\{0\}$.

Now assume that $S$ is PB (not necessarily smooth) and $\Phi$ is smooth. Then, $\Phi_{n}$ is continuous. If $S^{\prime}$ is a smooth real $(s, s)$-form cohomologous to $S$, we have

$$
\begin{aligned}
\left\langle S_{N}-S_{N}^{\prime}, \Phi\right\rangle & =\int\left[S-S^{\prime}\right] \wedge B_{N}+\frac{1}{N} \sum_{n=1}^{N} \frac{\left\langle S-S^{\prime}, \Phi_{n}\right\rangle}{n^{l_{s}-1} d_{s}^{n}} \\
& =\frac{1}{N} \sum_{n=1}^{N} \frac{\left\langle S-S^{\prime}, \Phi_{n}\right\rangle}{n^{l_{s}-1} d_{s}^{n}} .
\end{aligned}
$$

The last term tends to zero because $S-S^{\prime}$ is $\mathrm{PB}$ and $\left\|\Phi_{n}\right\|_{\mathrm{DSH}} \lesssim\left(d_{s}-\epsilon\right)^{n}$. It follows that $\left(S_{N}\right)$ converges to a $\mathrm{PB}$ current in $\mathbb{R} \Gamma_{s}$.

Let $R \in \mathbb{R} \Gamma_{s}$ be a current such that $[R]=0$. Then, using identity (3), we get

$$
|\langle R, \Phi\rangle|=d_{s}^{-n}\left|\left\langle\left(f^{n}\right)^{*} R, \Phi\right\rangle\right|=d_{s}^{-n}\left|\left\langle R, \Phi_{n}\right\rangle\right| \lesssim d_{s}^{-n}\left(d_{s}-\epsilon\right)^{n} .
$$


Therefore, $\langle R, \Phi\rangle=0$ and hence $R=0$. It follows that the restriction of [ ] to $\mathbb{R} \Gamma_{s}$ is injective.

Let $R \in \mathbb{R} \Gamma_{s}$ and let $\Phi$ be smooth. Using the identity $\left(f^{n}\right)^{*}[R]=d_{s}^{n}[R]$, we get

$$
\begin{aligned}
\langle R, \Phi\rangle & =d_{s}^{-n}\left\langle R,\left(f^{n}\right)_{*} \Phi\right\rangle \\
& =\int[R] \wedge\left(c_{0}+d_{s}^{-1} c_{1}+\cdots+d_{s}^{-n} c_{n}\right)+d_{s}^{-n}\left\langle R, \Phi_{n}\right\rangle .
\end{aligned}
$$

Since $R$ is $\mathrm{PB}$, when $n \rightarrow \infty$, we get $\langle R, \Phi\rangle=\int[R] \wedge c_{\Phi}$ with $c_{\Phi}:=\sum_{n \geq 0} d_{s}^{-n} c_{n}$. Following Proposition 2.1, $c_{\Phi}$ depends continuously on $\Phi$. Hence, we can extend $R$ to a continuous linear form on $\Phi \in \mathrm{DSH}^{k-s}(X)$ by

$$
\langle R, \Phi\rangle:=c_{\Phi}
$$

Hence $R$ is PC.

We show that the restriction of [] to $\mathbb{R C}(T)$ is injective. Let $R \in \mathbb{R C}(T)$ be a current such that $[R]=0$. We have to prove that $R=0$. We can write $R=R^{+}-R^{-}$ with $R^{ \pm}$positive closed currents such that $R^{ \pm} \leq c T$ for a constant $c>0$. Define $R_{n}^{ \pm}:=d_{s}^{n}\left(f^{n}\right)_{*} R^{ \pm}$and $R_{n}:=R_{n}^{+}-R_{n}^{-}$. We have $R_{n}^{ \pm} \leq c d_{s}^{n}\left(f^{n}\right)_{*} T=c T$. For a smooth test form $\Phi$, we have $\left|\left\langle T, \Phi_{n}^{ \pm}\right\rangle\right| \lesssim\left(d_{s}-\epsilon\right)^{n}$. The domination of $R_{n}^{ \pm}$and the negativity of $\Phi_{n}^{ \pm}$imply that $\left|\left\langle R_{n}, \Phi_{n}^{ \pm}\right\rangle\right| \lesssim\left(d_{s}-\epsilon\right)^{n}$. Since $\left[R_{n}\right]=0$, we obtain from (3) that

$$
|\langle R, \Phi\rangle|=d_{s}^{-n}\left|\left\langle\left(f^{n}\right)^{*} R_{n}, \Phi\right\rangle\right|=d_{s}^{-n}\left|\left\langle R_{n}, \Phi_{n}\right\rangle\right| \lesssim d_{s}^{-n}\left(d_{s}-\epsilon\right)^{n} .
$$

Hence $\langle R, \Phi\rangle=0$ and $R=0$. This completes the proof of Theorem 4.1.

Corollary 4.2. Let $f$ be a holomorphic automorphism of a compact Kähler manifold $X$ of dimension $k$. Assume that the dynamical degrees of $f$ are all distinct. Then for every $s, 1 \leq s \leq k$, there exists a non-zero PC positive closed $(s, s)$-current $T_{s}$ such that $f^{*} T_{s}=c_{s} T_{s}$ with $c_{s}>0$.

Proof. The hypothesis implies, thanks to the Khovanskii-Tessier-Gromov convexity theorem [31, 36, 27] (see Section 2.3), the existence of $m$ such that

$$
1<d_{1}<d_{2}<\cdots<d_{m}>d_{m+1}>\cdots>d_{k}=1 .
$$

Using Theorem 4.1, we construct the current $T_{s}$ such that $f^{*} T_{s}=d_{s} T_{s}$ for $1 \leq s \leq$ $m$. The current $T_{1}$ can be constructed as in Corollary 3.5. We now construct the other currents by induction using Theorem 3.1 for $f^{-1}$. We construct $(1,1)$-currents $S_{i}, 1 \leq i \leq k-m$, with Hölder continuous potentials and invariant currents $T_{s}$, $m+1 \leq s \leq k$, of the form $T_{s}=T_{m} \wedge S_{1} \wedge \ldots \wedge S_{s-m}$. These currents satisfy $f^{*} T_{s}=c_{s} T_{s}, c_{s}>0$. Since $f$ is an automorphism, we necessarily have $c_{k}=1$.

In order to apply inductively Theorem 3.1 for $f^{-1}$, we need only to verify that the first dynamical degree $\lambda_{1}\left(T_{s}\right)$ of $f^{-1}$, relative to $T_{s}$, is strictly larger than 1 for $m \leq s \leq k-1$. Following the last inequality of Proposition 2.6, it is sufficient to prove that $c_{s}>1$ for $m \leq s \leq k-1$. We have for every $\epsilon>0$

$$
\begin{aligned}
c_{s}^{-n} & \lesssim \int\left(f^{n}\right)_{*} T_{s} \wedge \omega^{k-s}=d_{m}^{-n} \int T_{m} \wedge\left(f^{n}\right)_{*}\left(S_{1} \wedge \ldots \wedge S_{s-m}\right) \wedge \omega^{k-s} \\
& =d_{m}^{-n} \int\left[S_{1}\right] \wedge \ldots \wedge\left[S_{s-m}\right] \wedge\left(f^{n}\right)^{*}\left[T_{m} \wedge \omega^{k-s}\right] \lesssim d_{m}^{-n}\left(d_{k-s+m}+\epsilon\right)^{n} .
\end{aligned}
$$

It follows that $c_{s}>1$ for $m \leq s \leq k-1$. This completes the induction step.

One can check that the wedge product of a PC positive closed current with a current of bidegree $(1,1)$ with a continuous potential is always PC. 


\section{Mixing OF THE EQUilibrium meAsure}

In this section, using the methods developed above, we can construct, for automorphisms with distinct dynamical degrees, an equilibrium measure which is PC and mixing. We get the following result.

Theorem 5.1. Let $f$ be a holomorphic automorphism of a compact Kähler manifold $X$ of dimension $k$. Assume that the dynamical degrees of $f$ are all distinct. Then $f$ admits a mixing PC invariant measure $\mu$. Moreover, $\mu$ gives no mass to sets with small Hausdorff dimension.

We need the following variation of Ahlfors's estimate (see [2] 35]).

Lemma 5.2. Let $f$ be a holomorphic automorphism of $X$. Let $T$ be a positive closed $(s, s)$-current. Assume that $\lambda_{1}(T):=\lim \left(\int\left(f^{n}\right)^{*} \omega \wedge T \wedge \omega^{k-s-1}\right)^{1 / n}<1$. Then for every smooth function $\psi \geq 0$, the limit values of the sequence $S_{n}:=\left(\psi \circ f^{n}\right) T$ are positive closed currents. Moreover, $\left\|\mathrm{d} S_{n}\right\| \rightarrow 0$ and $\left\|\mathrm{dd}^{\mathrm{c}} S_{n}\right\| \rightarrow 0$. If $S_{n_{i}} \rightarrow S$ and if $\sigma$ is a closed $(1,1)$-current with a continuous potential, then $S_{n_{i}} \wedge \sigma \rightarrow S \wedge \sigma$.

Proof. Let $\theta$ be a continuous $(0,1)$-form. The Cauchy-Schwarz inequality implies that

$$
\begin{aligned}
A_{n} & :=\left|\int\left(f^{n}\right)^{*}(\partial \psi) \wedge T \wedge \theta \wedge \omega^{k-s-1}\right| \\
& \leq\left|\int\left(f^{n}\right)^{*}(\partial \psi \wedge \overline{\partial \psi}) \wedge T \wedge \omega^{k-s-1}\right|^{1 / 2}\left|\int \theta \wedge \bar{\theta} \wedge T \wedge \omega^{k-s-1}\right|^{1 / 2} \\
& \leq c\left|\int\left(f^{n}\right)^{*} \omega \wedge T \wedge \omega^{k-s-1}\right|^{1 / 2}\left|\int \omega \wedge T \wedge \omega^{k-s-1}\right|^{1 / 2}
\end{aligned}
$$

if $i \partial \psi \wedge \overline{\partial \psi}$ and $i \theta \wedge \bar{\theta}$ are bounded by $c \omega, c>0$. It follows that $A_{n} \lesssim\left(\lambda_{1}(T)+\epsilon\right)^{n / 2}$. Since $\lambda_{1}(T)<1$, we have $\lim A_{n}=0$. As a consequence, $\lim \left\|\partial S_{n}\right\|=0$; hence $\lim \left\|\mathrm{d} S_{n}\right\|=0$.

To estimate $\left\|\operatorname{dd}^{\mathrm{c}} S_{n}\right\|$, we have just to observe that for $c>0$ large enough

$$
-c\left(f^{n}\right)^{*} \omega \wedge T \leq \operatorname{dd}^{\mathrm{c}}\left(f^{n}\right)^{*} \psi \wedge T \leq c\left(f^{n}\right)^{*} \omega \wedge T
$$

Let $u$ be a local continuous potential of $\sigma$ and let $\theta$ be a test form. Define $\psi_{n}:=\left(f^{n}\right)^{*} \psi$. For the last assertion of this lemma, we have

$$
\begin{aligned}
\left\langle\psi_{n} T \wedge \mathrm{dd}^{\mathrm{c}} u, \theta\right\rangle= & \left\langle\mathrm{dd}^{\mathrm{c}}(u T), \psi_{n} \theta\right\rangle \\
= & \left\langle\mathrm{dd}^{\mathrm{c}}\left(\psi_{n} T\right), u \theta\right\rangle+\left\langle\mathrm{d}\left(\psi_{n} T\right), u \mathrm{~d}^{\mathrm{c}} \theta\right\rangle \\
& -\left\langle\mathrm{d}^{\mathrm{c}}\left(\psi_{n} T\right), u \mathrm{~d} \theta\right\rangle+\left\langle\operatorname{dd}^{\mathrm{c}}\left(u \psi_{n} T\right), \theta\right\rangle .
\end{aligned}
$$

The first three terms tend to zero. Hence, $S_{n_{i}} \wedge \sigma \rightarrow S \wedge \sigma$.

Proof of Theorem 5.1. We construct invariant currents $T_{s}$ as in Corollary 4.2. We choose an extremal current $T_{m}$ in $\Gamma_{m}$. We can write $T_{s}=T_{m} \wedge S_{1} \wedge \ldots \wedge S_{s-m}$ for $s \geq m+1$, where $S_{i}$ are closed $(1,1)$-currents with Hölder continuous potentials. Define $\mu:=T_{k}$. Hence $\mu$ is PC. The estimate of the Hausdorff dimension of $\mu$ uses classical arguments [14 35. If the potentials of $S_{j}, 1 \leq j \leq k-m$, are $\alpha_{j}$-Hölder continuous, then $\mu$ gives no mass to sets whose Hausdorff dimension is smaller than $\alpha_{1}+\cdots+\alpha_{k-m}$.

We show first that $\mu$ is ergodic. Let $\psi \geq 0$ be a smooth test function. Let $\tau$ be the limit of a sequence of currents $n_{i}^{-1} \sum_{j=1}^{n_{i}}\left(\psi \circ f^{j}\right) T_{m}$. It is clear that $f^{*} \tau=d_{m} \tau$ 
and $\tau \leq\|\psi\|_{\infty} T_{m}$. Lemma 5.2 implies that $\tau$ is closed. Hence $\tau \in \Gamma_{m}$. Since $T_{m}$ is extremal in $\Gamma_{m}$, we have $\tau=c T_{m}$ for a constant $c$. We can now apply inductively Lemma 5.2. Since the currents $S_{r}$ have continuous potentials and $\lambda_{1}\left(T_{s}\right)<1$ for $m \leq s \leq k-1, n_{i}^{-1} \sum_{j=1}^{n_{i}}\left(\psi \circ f^{j}\right) \mu$ converge to $c \mu$. The invariance property of $\mu$ implies that $c=\|\mu\|^{-1} \int \psi \mathrm{d} \mu$. This constant does not depend on the sequence $\left(n_{i}\right)$. Consequently, $n^{-1} \sum_{j=1}^{n}\left(\psi \circ f^{j}\right) \mu$ converge to $c \mu$. Hence $\mu$ is ergodic.

We now prove that $\mu$ is mixing, which means $\left(f^{n}\right)^{*} \psi \mu \rightarrow c \mu, c=\|\mu\|^{-1} \int \psi \mathrm{d} \mu$, for every smooth function $\psi$. Let $M$ denote the set of measures which are limit values of the sequence $\left(f^{n *} \psi\right) \mu$ for some smooth function $\psi$. Since $\mathcal{C}\left(T_{m}\right)$ is finite dimensional, Lemma 5.2 implies that $M$ is a finite dimensional space which contains $\mu$ and which is invariant under $f^{*}$ and $f_{*}$. Let $E$ denote the space of functions $\varphi \in \mathrm{L}^{2}(\mu)$ such that $\int \varphi \mathrm{d} \mu^{\prime}=0$ for every $\mu^{\prime} \in M$ and let $E^{\perp}$ denote its orthogonal. Observe that these spaces are invariant under $f^{*}, f_{*}$ and that we have $\operatorname{dim} E^{\perp}=$ $\operatorname{dim} M$. Moreover, in $E$, every function can be approximated by smooth ones.

We show that $\operatorname{dim} E^{\perp}=1$. Since $f^{*}$ and $f_{*}$ preserve the scalar product in $\mathrm{L}^{2}(\mu)$, every eigenvalue of $f^{*}$ or $f_{*}$ has modulus equal to 1 . Let $\varphi$ be an eigenvector of $f_{*}$ associated to an eigenvalue $\lambda$. We have $f_{*}|\varphi|=|\varphi|$. The ergodicity of $\mu$ implies that $|\varphi|$ is constant. In particular, $\varphi^{n} \in \mathrm{L}^{2}(\mu)$ for every $n \geq 1$ and we have $f_{*} \varphi^{n}=\lambda^{n} \varphi^{n}$. We claim that $E$ does not contain any eigenvector. Otherwise, there is a function $\varphi \in E \backslash\{0\}$ such that $f_{*} \varphi=\lambda \varphi$ with a $\lambda$ such that $|\lambda|=1$. We have for every smooth function $\psi$

$$
|\langle\varphi \mu, \psi\rangle|=\left|\left\langle\left(f^{n}\right)_{*} \varphi \mu, \psi\right\rangle\right|=\left|\left\langle\left(f^{n *} \psi\right) \mu, \varphi\right\rangle\right| \rightarrow 0 .
$$

The last relation follows from the definition of $E$. We need of course to approach $\varphi$ by smooth functions in $E$. We get that $\varphi \mu=0$; hence $\varphi=0$. A contradiction.

Let $\varphi$ be an eigenvector of $f_{*}$ in $E^{\perp}$ associated to an eigenvalue $\lambda$. Then, $\varphi^{n}$ belongs to $E^{\perp}$ and is an eigenvector associated to $\lambda^{n}$ for every $n \geq 1$. Since $\operatorname{dim} E^{\perp}$ is finite, $\lambda$ is a root of unity. We have $f^{n *} \varphi=\varphi$ for some $n \geq 1$. Since $\mu$ is ergodic, $\varphi$ is constant. Hence, $\lambda=1$. Since $f_{\mid E^{\perp}}^{*}$ preserves the scalar product, $\operatorname{dim} E^{\perp}=1$ and $M$ is generated only by $\mu$. If $\left(f^{n_{i *}} \psi\right) \mu \rightarrow c \mu$, we have $c=\|\mu\|^{-1} \int \psi \mathrm{d} \mu$. This constant does not depend on $\left(n_{i}\right)$. Hence $\left(f^{n *} \psi\right) \mu \rightarrow c \mu$ and $\mu$ is mixing.

\section{REFERENCES}

[1] E. Bedford, M. Lyubich and J. Smillie, Polynomial diffeomorphisms of $\mathbb{C}^{2}$ IV: The measure of maximal entropy and laminar currents, Invent. Math., 112 (1993), 77-125. MR1207478 (94g:32035)

[2] E. Bedford and J. Smillie, Polynomial diffeomorphisms of $\mathbb{C}^{2}$ III: Ergodicity, exponents and entropy of the equilibrium measure, Math. Ann., 294 (1992), 395-420. MR1188127 (93k:32062)

[3] A. Blanchard, Sur les variétés analytiques complexes, Ann. Sci. Ecole Norm. Sup. (3), 73 (1956), 157-202. MR0087184 (19:316e)

[4] J.-B. Bost, H. Gillet, C. Soulé, Heights of projective varieties and positive Green forms, J. Amer. Math. Soc., 7 (1994), no. 4, 903-1027. MR1260106 (95j:14025)

[5] J.Y. Briend and J. Duval, Deux caractérisations de la mesure d'équilibre d'un endomorphisme de $\mathbb{P}^{k}(\mathbb{C})$, IHES Publ. Math., 93 (2001), 145-159. MR.1863737|(2002k:32027)

[6] S. Cantat, Dynamique des automorphismes des surfaces K3, Acta Math., 187 (2001), no. 1, 1-57. MR1864630 (2003h:32026)

[7] L. Clozel and E. Ullmo, Correspondances modulaires et mesures invariantes, J. reine angew. Math., 558 (2003), 47-83. MR.1979182 
[8] J.P. Demailly, Monge-Ampère Operators, Lelong numbers and Intersection theory in Complex Analysis and Geometry, Plenum Press (1993), 115-193 (V. Ancona and A. Silva editors). MR $1211880(94 \mathrm{k}: 32009)$

[9] J.P. Demailly, Introduction à la théorie de Hodge, Panoramas and Synthèses, 3 (1996), 1-111. MR 1409819

[10] J.P. Demailly, Pseudoconvex-concave duality and regularization of currents. Several complex variables (Berkeley, CA, 1995-1996), 233-271, Math. Sci. Res. Inst. Publ., 37, Cambridge Univ. Press, Cambridge, 1999. MR,1748605 (2002e:32046)

[11] T.C. Dinh, Distribution des préimages et des points périodiques d'une correspondance polynomiale, Bull. Soc. Math. France, to appear.

[12] T.C. Dinh, Suites d'applications méromorphes multivaluées et courants laminaires, preprint, 2003. arXiv:math.DS/0309421.

[13] T.C. Dinh and N. Sibony, Dynamique des applications d'allure polynomiale, Journal de Math. Pures et Appl., 82 (2003), 367-423. MR1992375 (2004e:37063)

[14] T.C. Dinh and N. Sibony, Dynamique des applications polynomiales semi-régulières, Arkiv för Matematik, 42 (2004), 61-85. MR2056545

[15] T.C. Dinh and N. Sibony, Groupes commutatifs d'automorphismes d'une variété kählérienne compacte, Duke Math. J., 123 (2004), no. 2, 311-328. MR2066940

[16] T.C. Dinh and N. Sibony, Distribution de valeurs d'une suite de transformations méromorphes et applications, preprint, 2003. arXiv:math.DS/0306095.

[17] T.C. Dinh and N. Sibony, Une borne supérieure de l'entropie topologique d'une application rationnelle, Ann. of Math., to appear.

[18] T.C. Dinh and N. Sibony, Regularization of currents and entropy, Ann. Sci. Ecole Norm. Sup., to appear.

[19] T.C. Dinh and N. Sibony, Dynamics of regular birational maps in $\mathbb{P}^{k}$, J. Funct. Anal., to appear.

[20] T.C. Dinh and N. Sibony, Decay of correlations and central limit theorem for meromorphic maps, preprint, 2004. arXiv:math.DS/0410008.

[21] C. Favre and V. Guedj, Dynamique des applications rationnelles des espaces multiprojectifs, Indiana Univ. Math. J., 50 (2001), no. 2, 881-934. MR1871393(2002m:32024)

[22] J.E. Forncess and N. Sibony, Complex Hénon mappings in $\mathbb{C}^{k}$ and Fatou-Biebebach domains, Duke Math. J., 65 (1992), 245-250. MR1150591(93d:32040)

[23] J.E. Forncess and N. Sibony, Complex dynamics in higher dimension, in Complex potential theory (Montréal, PQ, 1993), Nato ASI series Math. and Phys. Sci., vol. C439, Kluwer (1994), 131-186. MR.1332961 (96k:32057)

[24] H. Gillet and C. Soulé, Arithmetic intersection theory, I.H.E.S. Publ. Math., 72 (1990), 93-174 (1991). MR1087394 (92d:14016)

[25] P. Griffiths and J. Harris, Principles of algebraic geometry, Wiley Classics Library, John Wiley \& Sons, Inc., New York, 1994. MR1288523 (95d:14001)

[26] M. Gromov, On the entropy of holomorphic maps, Enseignement Math., 49 (2003), 217-235 (manuscript, 1977). MR2026895

[27] M. Gromov, Convex sets and Kähler manifolds, Advances in differential geometry and topology, Word Sci. Publishing, Teaneck, NJ, 1998, 1-38. MF,1095529 (92d:52018)

[28] V. Guedj, Dynamics of polynomial mappings of $\mathbb{C}^{2}$, Amer. J. Math., 124 (2002), no. 1, 75-106. MR1879000 (2003b:32021)

[29] $V$. Guedj, Ergodic properties of rational mappings with large topological degree, Ann. of Math., to appear.

[30] V. Guedj and N. Sibony, Dynamics of polynomial automorphisms of $\mathbb{C}^{k}$, Arkiv för Matematik, 40 (2002), 207-243. MR1948064 (2004b:32029)

[31] A. Khovanskii, Fewnomials and Pfaff manifolds, I.C.M. 1983, Warsawa (1984), 549-565. MR 0804711 (87d:58014)

[32] G. A. Margulis, Discrete subgroups of semisimple Lie groups, Springer-Verlag, 1991. MR $1090825(92 \mathrm{~h}: 22021)$

[33] B. Mazur, The topology of rational points, Experiment Math., 1 (1992), 35-45. MR1181085 (93j:14020)

[34] C.T. McMullen, Dynamics on K3 surfaces: Salem numbers and Siegel disks, J. reine angew. Math., 545 (2002), 201-233. MR 1896103 (2003a:37057) 
[35] N. Sibony, Dynamique des applications rationnelles de $\mathbb{P}^{k}$, Panoramas et Synthèses, 8 (1999), 97-185. MR,1760844 (2001e:32026)

[36] B. Tessier, Bonnesen-type inequalities in algebraic geometry, Sem. on Diff. Geom. (1982), 85-105. Princeton Univ. Press. MR0645731 (83d:52010)

[37] C. Voisin, Intrinsic pseudovolume forms and K-correspondences, preprint, 2003, arXiv: math.AG/0212110.

[38] Y. Yomdin, Volume growth and entropy, Israel J. Math., 57 (1987), 285-300. MR0889979 (90g:58008)

Mathématique - BÂt. 425, UMR 8628, Université Paris-Sud, 91405 Orsay, France

E-mail address: TienCuong.Dinh@math.u-psud.fr

Mathématique - BÂt. 425, UMR 8628, Université Paris-Sud, 91405 Orsay, France

E-mail address: Nessim.Sibony@math.u-psud.fr 